TRANSACTIONS OF THE

AMERICAN MATHEMATICAL SOCIETY

Volume 357, Number 3, Pages 1245-1274

S 0002-9947(04)03568-8

Article electronically published on September 2, 2004

\title{
BROWNIAN MOTION IN TWISTED DOMAINS
}

\author{
DANTE DEBLASSIE AND ROBERT SMITS
}

\begin{abstract}
The tail behavior of a Brownian motion's exit time from an unbounded domain depends upon the growth of the "inner radius" of the domain. In this article we quantify this idea by introducing the notion of a twisted domain in the plane. Roughly speaking, such a domain is generated by a planar curve as follows. As a traveler proceeds out along the curve, the boundary curves of the domain are obtained by moving out $\pm g(r)$ units along the unit normal to the curve when the traveler is $r$ units away from the origin. The function $g$ is called the growth radius. Such domains can be highly nonconvex and asymmetric. We give a detailed account of the case $g(r)=\gamma r^{p}, 0<p \leq 1$. When $p=1$, a twisted domain can reasonably be interpreted as a "twisted cone."
\end{abstract}

\section{INTRODUCTION}

Let $G$ be the planar domain lying above the parabola $y=A x^{2}, A>0$. In Bañuelos, DeBlassie and Smits (2001) it was shown that the exit time $\tau_{G}$ of Brownian motion from $G$ has unusual behavior: for some positive constants $A_{1}$ and $A_{2}$,

$$
-A_{1} \leq \liminf _{t \rightarrow \infty} t^{-1 / 3} \log P\left(\tau_{G}>t\right) \leq \limsup _{t \rightarrow \infty} t^{-1 / 3} \log P\left(\tau_{G}>t\right) \leq-A_{2} .
$$

Though not stated in that article, the method extends to more general domains $\left\{(x, y): y>A x^{q}\right\}, A>0, q>1$ yielding an analog of (1.0) where $t^{-1 / 3}$ is replaced by $t^{-(q-1) /(q+1)}$.

Using Gaussian techniques, Li (2002) extended (1.0) to regions $G=\{(x, y) \in$ $\left.\mathbb{R}^{d+1}: y>A\|x\|^{q}, x \in \mathbb{R}^{d}\right\}, A>0, q>1, d \geq 2$, giving the constants $A_{1}$ and $A_{2}$ explicitly in terms of zeros of Bessel functions. He also obtained estimates for more general unbounded regions. Lifshits and Shi (2002) extended Li's results, explicitly identifying

$$
\lim _{t \rightarrow \infty} t^{-(q-1) /(q+1)} \log P\left(\tau_{G}>t\right)
$$

in terms of zeros of Bessel functions. M. van den Berg (2003) has obtained analogs of these results for the heat kernel.

All these articles exploit the symmetry and convexity of the unbounded region $G$. Since the power of $t$ multiplying $\log P\left(\tau_{G}>t\right)$ is independent of the dimension, it seems the asymptotic behavior of $\log P\left(\tau_{G}>t\right)$ is essentially determined by the rate at which the domain "expands" along the axis of symmetry as a traveler on the axis moves toward infinity. That is, at distance $y$ from the origin along the axis of symmetry, the "width" of $G$ is $2 y^{1 / q}$. The key here is that "width" is measured

Received by the editors November 5, 2002 and, in revised form, November 3, 2003.

2000 Mathematics Subject Classification. Primary 60J65, 60J50, 60F10.

Key words and phrases. Exit times, Brownian motion, twisted domains. 


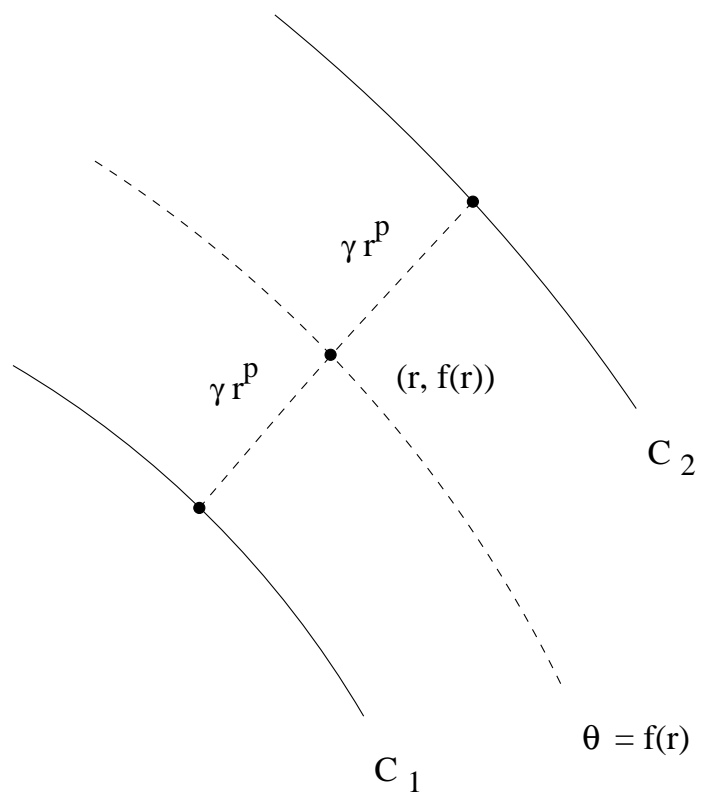

FiguRE 1.

perpendicular to some curve lying within the region. The purpose of this article is to describe the connection between the growth rate of the "inner radius" of the domain and the rate of decay of the tail distribution of the exit time of Brownian motion from the domain.

These considerations motivate the following definition of a twisted domain. For simplicity, we restrict attention to planar domains.

Let $D \subseteq \mathbb{R}^{2}$ be a domain whose boundary consists of three curves (in polar coordinates)

$$
\begin{aligned}
& C_{1}: \theta=f_{1}(r), \quad r \geq r_{1}, \\
& C_{2}: \theta=f_{2}(r), \quad r \geq r_{1}, \\
& C_{3}: \quad r=r_{1}, \quad f_{2}\left(r_{1}\right) \leq \theta \leq f_{1}\left(r_{1}\right),
\end{aligned}
$$

where $f_{1}$ and $f_{2}$ are smooth and the curves $C_{1}$ and $C_{2}$ do not cross:

$$
0<f_{1}(r)-f_{2}(r)<2 \pi, \quad r \geq r_{1} .
$$

We say $D$ is a twisted domain if there are constants $r_{0}>0, \gamma>0$ and $p \in(0,1]$ and a smooth function $f(r)$ such that the curves $f_{1}(r)$ and $f_{2}(r), r \geq r_{0}$, are obtained from $f(r)$ by moving out $\pm \gamma r^{p}$ units along the normal to the curve $\theta=f(r)$ at the point whose polar coordinates are $(r, f(r))$. We call $\gamma r^{p}$ the growth radius and $\theta=f(r)$ the generating curve. See Figure 1 .

There is a question of consistency: the generating curve must yield nonintersecting boundary curves that can be parameterized by distance to the origin. To ensure consistency, we make the following hypotheses on the generating curve:

$$
\begin{aligned}
& r f^{\prime}(r) \rightarrow 0 \quad \text { as } \quad r \rightarrow \infty, \\
& r\left(r f^{\prime}(r)\right)^{\prime} \rightarrow 0 \text { as } \quad r \rightarrow \infty .
\end{aligned}
$$


These hypotheses arise naturally to permit parameterization of the boundary curves by distance to the origin. The condition (1.1) will also ensure that the curves generated by $f(r)$ do not cross. In fact, when $f(r) \uparrow \infty$ as $r \uparrow \infty$ (a spiral), it is possible to show that $r f^{\prime}(r) \rightarrow \infty$ as $r \rightarrow \infty$ would force the generated curves to cross. Intuitively, the spiral $\theta=f(r)$ does not have "enough spacing" between successive journeys about the origin.

Note that by (1.1) and (1.2) the generating curve really comes into play only for parts of the domain far away from the origin. Stated another way, the asymptotics in Theorems 1.1 and 1.2 below are unchanged if a compact set is adjoined to the twisted domain $D$. Also observe that twisted domains can be nonsymmetric and/or nonconvex. Now we state our main theorems. In what follows, for $0<p<1$ and $b>0$ set

$$
C_{p, b}=(1+p)\left[\frac{\pi^{2+p}}{8^{p} p^{2 p} b^{2}(1-p)^{1-p}} \frac{\Gamma^{2 p}\left(\frac{1-p}{2 p}\right)}{\Gamma^{2 p}\left(\frac{1}{2 p}\right)}\right]^{\frac{1}{p+1}} .
$$

Theorem 1.1. Let $D \subseteq \mathbb{R}^{2}$ be a twisted domain with growth radius $\gamma r^{p}, \gamma>0$, $0<p<1$. Then

$$
\lim _{t \rightarrow \infty} t^{-\frac{1-p}{1+p}} \log P_{z}\left(\tau_{D}>t\right)=\left[\frac{\pi^{2 p-1}}{\gamma 2^{2 p}(1-p)^{2 p}}\right]^{\frac{2}{1+p}} C_{p, 1} .
$$

Theorem 1.2. Let $D \subseteq \mathbb{R}^{2}$ be a twisted domain with growth radius $\gamma r, \gamma>0$. Then

$$
\lim _{t \rightarrow \infty}[\log t]^{-1} \log P_{z}\left(\tau_{D}>t\right)=\pi\left[4 \arccos \frac{1}{\sqrt{1+\gamma^{2}}}\right]^{-1} .
$$

Remark. Using Theorem 1 from van den Berg (2003), our results imply that the heat kernel $p_{D}(t, x, y)$ for $D$ in our Theorem 1.1 satisfies

$$
\lim _{t \rightarrow \infty} t^{-\frac{1-p}{1+p}} \log p_{D}(t, x, y)=-\left[\frac{\pi^{2 p-1}}{\gamma 2^{p}(1-p)^{2 p}}\right]^{\frac{2}{1+p}} C_{p, 1} .
$$

The article is organized as follows. In sections 2 and 3 we show that our hypotheses (1.1) and (1.2) prevent inconsistencies: distance to the origin $r$ can be used as a parameter and the boundary curves do not cross. In section 4 we collect some results on conformal maps. Functionals of Brownian motion in a strip comprise the object of study in section 5 . In section 6 we derive properties of conformal maps from $D$ into strips. Section 7 is the place we prove our main results, and section 8 gives the proof of a technical result from section 6 .

We close this introduction with several examples and remarks.

Example 1. If the generating curve is $\theta \equiv$ constant and $p=1$, then $D$ is a cone and Theorem 1.2 reduces to a weak version of an old result of Spitzer (1958). In light of this, it is reasonable to call any twisted domain with $p=1$ a twisted cone.

Example 2. Let $g(x)$ satisfy

$$
g(x) \rightarrow L \in[0, \infty], \quad g^{\prime}(x) \rightarrow 0, \quad x g^{\prime \prime}(x) \rightarrow 0,
$$

all as $x \rightarrow \infty$. Then $y=g(x)$ can be represented in polar coordinates as $\theta=f(r)$, and we get a generating curve satisfying (1.1) and (1.2). Our theorems apply for growth radius $\gamma r^{p}, 0<p \leq 1$. 
For the special case $g \equiv 0$ and $0<p<1$, the corresponding twisted domain is

$$
\left\{(x, y):|y|<\gamma|x|^{p}\right\}
$$

which is a rotation of the domain

$$
\widetilde{D}=\left\{(x, y): y>\gamma^{-1 / p}|x|^{1 / p}\right\}
$$

considered by Li (2002) and Lifshits and Shi (2002).

Example 3. Spirals. If the generating curve is given by

$$
\theta=(\ln r)^{q},
$$

where $0<q<1$, then the hypotheses (1.1) and (1.2) hold and our theorems apply.

Example 4. Oscillation at infinity. Let the generating curve be given by

$$
\theta=\sin \left((\ln r)^{q}\right)
$$

where $0<q<1$. Hypotheses (1.1) and (1.2) hold and our theorems apply. Notice that the domain "oscillates at $\infty$ ", in contrast to the previous example, where the domain spins round and round.

Example 5. Consider the domain

$$
D=\left\{(x, y): y>|x|^{q} \text { for } x \leq 0 \text { and } y>|x|^{p} \text { for } x>0\right\},
$$

where $1<q<p$. Then our theorems show that the $|x|^{q}$ part of the boundary determines the asymptotic behavior: for some positive $A=A(q)$,

$$
\lim _{t \rightarrow \infty} t^{-(q-1) /(q+1)} \log P\left(\tau_{D}>t\right)=A .
$$

Rather than give the rigorous details, we present a plausibility argument. In the rest of this example, we use the notation $g \approx h$ to mean " $g$ is approximately $h$ " in an intuitive sense. Since the normal to the boundary of $D$ converges to a vertical line at $\infty$, the "halfway curve"

$$
x=\frac{1}{2}\left[y^{1 / p}-y^{1 / q}\right]
$$

is more or less a generating curve for $D$.

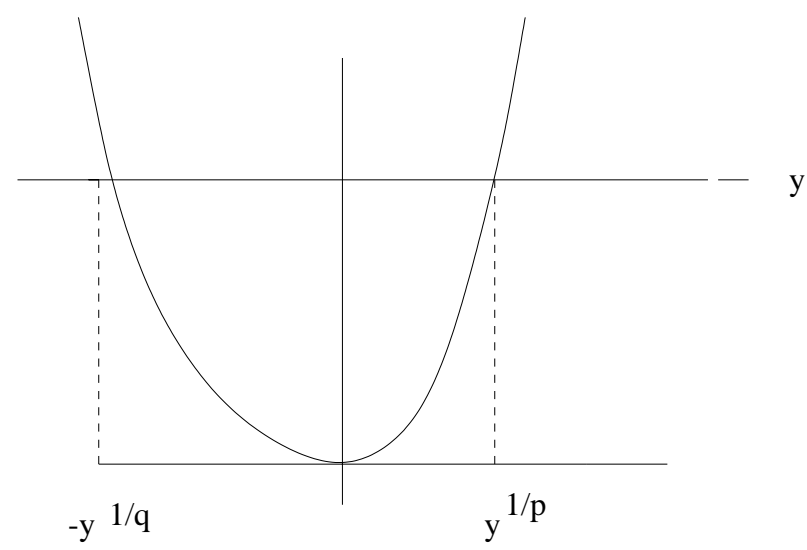

Moreover, the growth radius at the level $y$ is about

$$
\frac{1}{2}\left[y^{1 / p}+y^{1 / q}\right] \approx \frac{1}{2} y^{1 / q}, \quad \text { for large } y,
$$


since $1<q<p$. The angular coordinate of a point on the growth curve is

$$
\begin{aligned}
\theta & =\pi+\tan ^{-1} \frac{2 y}{y^{1 / p}-y^{1 / q}} \\
& \approx \pi-\tan ^{-1} 2 y^{1-\frac{1}{q}}, \text { for large } y,
\end{aligned}
$$

and the corresponding distance to the origin is

$$
\begin{aligned}
r & =\sqrt{y^{2}+\frac{1}{4}\left[y^{1 / p}-y^{1 / q}\right]^{2}} \\
& \approx y, \text { for large } y .
\end{aligned}
$$

Thus our generating curve has the polar form

$$
\theta=\pi-\tan ^{-1} 2 r^{1-1 / q},
$$

and it is easy to verify that (1.1) and (1.2) hold. Since the growth radius is about $\frac{1}{2} y^{1 / q} \approx \frac{1}{2} r^{1 / q}$, we get the asserted asymptotic behavior. One can make this argument rigorous by showing that the twisted domains

$$
\begin{aligned}
& D_{1}: \text { growth curve } \theta=\pi-\tan ^{-1} 2 r^{1-1 / q}, \text { growth radius }\left(\frac{1}{2}-\varepsilon\right) r^{1 / q}, \\
& D_{2}: \text { growth curve } \theta=\pi-\tan ^{-1} 2 r^{1-1 / q}, \text { growth radius }\left(\frac{1}{2}+\varepsilon\right) r^{1 / q},
\end{aligned}
$$

satisfy

$$
D_{1} \subseteq D \subseteq D_{2}
$$

\section{Consistency: Parameterization By $r$}

Our setup involving the growth radius $\gamma r^{p}$ leads to a natural parameterization of the boundary curves in terms of the distance $t$ from the origin to the point corresponding to $\theta=f(t)$ on the generating curve. Here are the details.

Let $P=(t \cos f(t), t \sin f(t))$ be the Cartesian coordinates of a point on the generating curve, and suppose $Q_{1}$ and $Q_{2}$ are the points on $C_{1}$ and $C_{2}$, respectively, that are $\mp \gamma t^{p}$ units along the normal at $P$. The unit tangent vector at $P$ is

$$
\frac{\left(\cos f-t f^{\prime} \sin f, \sin f+t f^{\prime} \cos f\right)}{\sqrt{1+\left(t f^{\prime}\right)^{2}}},
$$

and the unit normal at $P$ (obtained by rotating the unit tangent $90^{\circ}$ clockwise) is

$$
\vec{N}=\vec{N}(t)=\frac{\left(\sin f+t f^{\prime} \cos f,-\cos f+t f^{\prime} \sin f\right)}{\sqrt{1+\left(t f^{\prime}\right)^{2}}} .
$$

Thus

$$
\begin{aligned}
\overrightarrow{O Q_{1}} & =\overrightarrow{O P}+\overrightarrow{P Q_{1}} \\
& =\overrightarrow{O P}-\gamma t^{p} \vec{N}
\end{aligned}
$$

This yields the following parametric representation of $C_{1}$ :

$$
\begin{aligned}
& x_{1}(t)=t \cos f(t)-\frac{\gamma t^{p}\left[\sin f(t)+t f^{\prime}(t) \cos f(t)\right]}{\sqrt{1+\left[t f^{\prime}(t)\right]^{2}}}, \\
& y_{1}(t)=t \sin f(t)-\frac{\gamma t^{p}\left[-\cos f(t)+t f^{\prime}(t) \sin f(t)\right]}{\sqrt{1+\left[t f^{\prime}(t)\right]^{2}}} .
\end{aligned}
$$


Similarly, $C_{2}$ is given by

$$
\begin{aligned}
& x_{2}(t)=t \cos f(t)+\frac{\gamma t^{p}\left[\sin f(t)+t f^{\prime}(t) \cos f(t)\right]}{\sqrt{1+\left[t f^{\prime}(t)\right]^{2}}}, \\
& y_{2}(t)=t \sin f(t)+\frac{\gamma t^{p}\left[-\cos f(t)+t f^{\prime}(t) \sin f(t)\right]}{\sqrt{1+\left[t f^{\prime}(t)\right]^{2}}} .
\end{aligned}
$$

Denote by $r_{i}(t)$ and $\theta_{i}(t)$ the polar coordinates of $Q_{i}$. Then for

$$
h(t)=\frac{t^{p} f^{\prime}(t)}{\sqrt{1+\left[t f^{\prime}(t)\right]^{2}}},
$$

we have

$$
\begin{aligned}
& r_{1}^{2}(t)=t^{2}\left[1+\gamma^{2} t^{2 p-2}-2 \gamma h(t)\right], \\
& r_{2}^{2}(t)=t^{2}\left[1+\gamma^{2} t^{2 p-2}+2 \gamma h(t)\right] .
\end{aligned}
$$

Moreover, using that the angle between the position vector $\overrightarrow{O P}$ and the tangent vector at $P$ is no more than $90^{\circ}$ (their dot product is nonnegative), we have

$$
\begin{aligned}
& \theta_{1}(t)=f(t)+\angle\left(\overrightarrow{O P}, \overrightarrow{O Q_{1}}\right), \\
& \theta_{2}(t)=f(t)-\angle\left(\overrightarrow{O P}, \overrightarrow{O Q_{2}}\right),
\end{aligned}
$$

where

$$
\angle\left(\overrightarrow{O P}, \overrightarrow{O Q}_{i}\right)=\arccos \frac{\overrightarrow{O P} \cdot \overrightarrow{O Q_{i}}}{|\overrightarrow{O P}|\left|\overrightarrow{O Q_{i}}\right|}, \quad i=1,2 .
$$

Upon computing the latter and substituting into the equations for $\theta_{i}$, we get

$$
\begin{aligned}
& \theta_{1}(t)=f(t)+\arccos \frac{1-\gamma h(t)}{\sqrt{1+\gamma^{2} t^{2 p-2}-2 \gamma h(t)}}, \\
& \theta_{2}(t)=f(t)-\arccos \frac{1+\gamma h(t)}{\sqrt{1+\gamma^{2} t^{2 p-2}+2 \gamma h(t)}} .
\end{aligned}
$$

The main result of this section is the next theorem.

Theorem 2.1. For $i=1,2, r_{i}(t)$ is increasing for large $t$.

Proof. Differentiation of 2.2) yields

$$
2 r_{1} r_{1}^{\prime}=2 t\left[1+p \gamma^{2} t^{2 p-2}-2 \gamma h-\gamma t h^{\prime}\right] .
$$

Since $\left(t f^{\prime}\right)^{\prime}=f^{\prime}+t f^{\prime \prime}$, differentiation of (2.1) gives

$$
\begin{aligned}
h^{\prime} & =\frac{t^{p-1}\left[p f^{\prime}+(p-1) t^{2}\left(f^{\prime}\right)^{3}+t f^{\prime \prime}\right]}{\left(\sqrt{1+\left(t f^{\prime}\right)^{2}}\right)^{3}} \\
& =\frac{t^{p-1}\left[(p-1) f^{\prime}+(p-1) t^{2}\left(f^{\prime}\right)^{3}+\left(t f^{\prime}\right)^{\prime}\right]}{\left(\sqrt{1+\left(t f^{\prime}\right)^{2}}\right)^{3}} .
\end{aligned}
$$

Hypothesis (1.1) forces $t^{p} f^{\prime}(t) \rightarrow 0$ as $t \rightarrow \infty$, and so

$$
h(t) \rightarrow 0 \quad \text { as } \quad t \rightarrow \infty .
$$

By hypotheses (1.1) and (1.2),

$$
t h^{\prime}(t) \rightarrow 0 \quad \text { as } \quad t \rightarrow \infty .
$$


In fact,

$$
t^{1-p} h(t) \rightarrow 0 \quad \text { as } \quad t \rightarrow \infty
$$

By (2.7) and (2.8)

$$
1+p \gamma^{2} t^{2 p-2}-2 \gamma h(t)-\gamma t h^{\prime}(t) \rightarrow \begin{cases}1+\gamma^{2}, & p=1, \\ 1, & p<1 .\end{cases}
$$

In any event, we see from (2.6) that $r_{1}^{\prime}(t)>0$ for large $t$. The argument for $r_{2}$ is similar.

Corollary 2.2. For $i=1,2$,

$$
\lim _{t \rightarrow \infty} \frac{r_{i}(t)}{t_{i}^{\prime}(t)}=1
$$

Proof. For $i=1$, we have

$$
\begin{aligned}
\frac{r_{1}(t)}{t r_{1}^{\prime}(t)} & =\frac{r_{1}^{2}(t)}{t r_{1}(t) r_{1}^{\prime}(t)} \\
& =\frac{1+\gamma^{2} t^{2 p-2}-2 \gamma h}{1+p \gamma^{2} t^{2 p-2}-2 \gamma h-\gamma t h^{\prime}} \\
& \rightarrow 1 \quad \text { as } \quad t \rightarrow \infty
\end{aligned}
$$

by (2.7) and (2.10). The case $i=2$ is similar.

An immediate consequence of this theorem is that the boundary curves arising from the generating function $f$ are indeed parameterizable by distance to the origin. In particular, we have (making $r_{0}$ large enough)

$$
C_{i}: f_{i}(r)=\theta_{i}\left(r_{i}^{-1}(r)\right), \quad r \geq r_{0}, \quad i=1,2 .
$$

\section{Consistency: The Boundary Curves do not Cross}

The main result of this section is the next theorem.

Theorem 3.1. As $r \rightarrow \infty$,

$$
\theta_{1}\left(r_{1}^{-1}(r)\right)-\theta_{2}\left(r_{2}^{-1}(r)\right) \sim \begin{cases}2 \gamma r^{p-1}, & p<1, \\ 2 \arccos \frac{1}{\sqrt{1+\gamma^{2}}}, & p=1 .\end{cases}
$$

Corollary 3.2. For large $r$,

$$
\theta_{1}\left(r_{1}^{-1}(r)\right)-\theta_{2}\left(r_{2}^{-1}(r)\right) \in(0,2 \pi) .
$$

By the corollary, the boundary curves do not cross and our setup is consistent, as claimed. To prove Theorem 3.1 we need the following four lemmas.

Lemma 3.3. For $i=1,2, t \theta_{i}^{\prime}(t) \rightarrow 0$ as $t \rightarrow \infty$.

Proof. With $h(t)$ from (2.1), write

$$
g(t)=\arccos \frac{1-\gamma h(t)}{\sqrt{1+\gamma^{2} t^{2 p-2}-2 \gamma h(t)}} .
$$

Then for $i=1$, by (2.4)

$$
\theta_{i}(t)=f(t)+g(t)
$$


Now

$$
t g^{\prime}(t)=\frac{\gamma\left[\gamma t^{2 p-2}-h(t)\right] t h^{\prime}(t)-(1-p) \gamma t^{2 p-2}[1-\gamma h(t)]}{\sqrt{t^{2 p-2}-h(t)^{2}}\left[1+\gamma^{2} t^{2 p-2}-2 \gamma h(t)\right]} .
$$

For $p=1, t g^{\prime}(t) \rightarrow 0$ as $t \rightarrow \infty$, by (2.7)-(2.8). By hypotheses, $t f^{\prime}(t) \rightarrow 0$ as $t \rightarrow \infty$, so the desired conclusion follows from (3.1).

If $p<1$, then rearrangement of $t g^{\prime}$ yields

$$
t g^{\prime}(t)=\frac{\gamma t^{p-1}}{\sqrt{t^{2 p-2}-h(t)^{2}}} \frac{\left[\gamma t^{p-1}-t^{1-p} h(t)\right] t h^{\prime}(t)-(1-p) t^{p-1}[1-\gamma h(t)]}{\left[1+\gamma^{2} t^{2 p-2}-2 \gamma h(t)\right]} .
$$

We have from (2.1)

$$
\begin{aligned}
t^{2 p-2}-h(t)^{2} & =t^{2 p-2}-\frac{t^{2 p}\left[f^{\prime}(t)\right]^{2}}{1+\left[t f^{\prime}(t)\right]^{2}} \\
& =\frac{t^{2 p-2}}{1+\left[t f^{\prime}(t)\right]^{2}} .
\end{aligned}
$$

Then, using (2.7) and (2.8),

$$
t g^{\prime}(t) \rightarrow 0 \quad \text { as } \quad t \rightarrow \infty
$$

Again we get the desired conclusion.

The case $i=2$ is similar.

Lemma 3.4. For $i=1,2$,

$$
\frac{\theta_{i}\left(r_{i}^{-1}\left(r_{2}\right)\right)-\theta_{i}\left(r_{i}^{-1}\left(r_{1}\right)\right)}{r_{2}-r_{1}} \rightarrow 0 \quad \text { as } \quad r_{1}, r_{2} \rightarrow \infty .
$$

Proof. For $i=1$, there is $\tilde{r}$ between $r_{1}$ and $r_{2}$ such that

$$
\begin{aligned}
\frac{\theta_{1}\left(r_{1}^{-1}\left(r_{2}\right)\right)-\theta_{1}\left(r_{1}^{-1}\left(r_{1}\right)\right)}{r_{2}-r_{1}} & =\theta_{1}^{\prime}\left(r_{1}^{-1}(\tilde{r})\right)\left(r_{1}^{-1}\right)^{\prime}(\tilde{r}) \\
& =r_{1}^{-1}(\tilde{r}) \theta_{1}^{\prime}\left(r_{1}^{-1}(\tilde{r})\right) \cdot \frac{1}{r_{1}^{-1}(\tilde{r}) r_{1}^{\prime}\left(r_{1}^{-1}(\tilde{r})\right)} .
\end{aligned}
$$

Since $\tilde{r} \rightarrow \infty$ as $r_{1}, r_{2} \rightarrow \infty$, it follows that $r_{1}^{-1}(\tilde{r}) \rightarrow \infty$, and by (2.2) and (2.6)

$$
r_{1}^{\prime}\left(r_{1}^{-1}(\tilde{r})\right) \rightarrow\left\{\begin{array}{cc}
\sqrt{1+\gamma^{2}}, & p=1 \\
1, & p<1 .
\end{array}\right.
$$

Hence by Lemma 3.3 we can let $r_{1}, r_{2} \rightarrow \infty$ in 3.3 to get the desired conclusion.

Lemma 3.5. As $t \rightarrow \infty$,

$$
\arccos \frac{1 \pm \gamma h(t)}{\sqrt{1+\gamma^{2} t^{2 p-2} \pm 2 \gamma h(t)}} \sim\left\{\begin{array}{cl}
\gamma t^{p-1}, & p<1 \\
\arccos \frac{1}{\sqrt{1+\gamma^{2}}}, & p=1 .
\end{array}\right.
$$

Proof. For $p=1$, this expression follows immediately from the fact that $h(t) \rightarrow 0$ (see (2.7)). By the same token, for $p<1$ the argument of the arccosine converges 
to 1 . Since $\arccos x \sim \sqrt{2} \sqrt{1-x}$ as $x \rightarrow 1$,

$$
\begin{aligned}
& {\left[\arccos \frac{1 \pm \gamma h(t)}{\sqrt{1+\gamma^{2} t^{2 p-2} \pm 2 \gamma h(t)}}\right]^{2} \sim 2\left[1-\frac{1 \pm \gamma h(t)}{\sqrt{1+\gamma^{2} t^{2 p-2} \pm 2 \gamma h(t)}}\right]} \\
& \quad=2 \frac{\gamma^{2}\left[t^{2 p-2}-h(t)^{2}\right]}{\sqrt{1+\gamma^{2} t^{2 p-2} \pm 2 \gamma h(t)}\left[\sqrt{1+\gamma^{2} t^{2 p-2} \pm 2 \gamma h(t)}+(1 \pm \gamma h(t))\right]} \\
& \quad \sim \gamma^{2}\left[t^{2 p-2}-h(t)^{2}\right] \quad(\text { since } h(t) \rightarrow 0) \\
& \quad=\gamma^{2}\left[t^{2 p-2}-\frac{t^{2 p}\left[f^{\prime}(t)\right]^{2}}{1+\left[t f^{\prime}(t)\right]^{2}}\right] \quad(\text { by (2.1) }) \\
& \quad=\frac{\gamma^{2} t^{2 p-2}}{1+\left[t f^{\prime}(t)\right]^{2}} \\
& \quad \sim \gamma^{2} t^{2 p-2},
\end{aligned}
$$

by our hypothesis that $t f^{\prime}(t) \rightarrow 0$.

Lemma 3.6. Let $t_{1}=t_{1}(r)$ and $t_{2}=t_{2}(r)$ satisfy $t_{1} \rightarrow \infty, t_{2} \rightarrow \infty, \frac{t_{1}}{t_{2}} \rightarrow L$, and $t_{1}^{2}-t_{2}^{2}=O\left(t_{2}^{p-1} t_{1}^{2}\right)$ as $r \rightarrow \infty$. Then

$$
\frac{f\left(t_{1}\right)-f\left(t_{2}\right)}{t_{2}^{p-1}} \rightarrow 0 \quad \text { as } \quad r \rightarrow \infty
$$

Proof. For some $\tilde{t}$ between $t_{1}$ and $t_{2}$,

$$
\begin{aligned}
\frac{f\left(t_{1}\right)-f\left(t_{2}\right)}{t_{2}^{p-1}} & =\frac{f^{\prime}(\tilde{t})\left(t_{1}-t_{2}\right)}{t_{2}^{p-1}} \\
& =\tilde{t} f^{\prime}(\tilde{t}) \cdot \frac{t_{1}^{2}-t_{2}^{2}}{t_{2}^{p-1}} \cdot \frac{1}{\tilde{t}\left(t_{1}+t_{2}\right)} .
\end{aligned}
$$

Since $\tilde{t} \rightarrow \infty$ as $r \rightarrow \infty, \tilde{t} f^{\prime}(\tilde{t}) \rightarrow 0$ as $r \rightarrow \infty$. Hence it suffices to show that

$$
\frac{t_{1}^{2}-t_{2}^{2}}{t_{2}^{p-1}} \cdot \frac{1}{\tilde{t}\left(t_{1}+t_{2}\right)} \text { is bounded as } r \rightarrow \infty
$$

Indeed, this quantity can be rewritten as

$$
\frac{t_{1}^{2}-t_{2}^{2}}{t_{1}^{2} t_{2}^{p-1}} \cdot \frac{t_{1}}{\tilde{t}} \cdot \frac{t_{1}}{t_{1}+t_{2}}
$$

and it is easy to see our hypotheses imply this is bounded as $r \rightarrow \infty$.

Proof of Theorem 3.1. Write $t_{i}=r_{i}^{-1}(r)$, so that we have

$$
r=r_{1}\left(t_{1}\right)=r_{2}\left(t_{2}\right) \text {. }
$$

By (2.2)-(2.3),

$$
\begin{aligned}
& t_{1}=r_{1}\left(t_{1}\right)\left[1+\gamma^{2} t_{1}^{2 p-2}-2 \gamma h\left(t_{1}\right)\right]^{-1 / 2} \\
& t_{2}=r_{2}\left(t_{2}\right)\left[1+\gamma^{2} t_{2}^{2 p-2}+2 \gamma h\left(t_{2}\right)\right]^{-1 / 2}
\end{aligned}
$$

Then (3.4) forces

$$
\frac{t_{1}}{t_{2}} \rightarrow 1 \quad \text { as } \quad r \rightarrow \infty
$$


By (3.4)-(3.6),

$$
t_{1}^{2}-t_{2}^{2}=r_{1}^{2}\left(t_{1}\right) \frac{\gamma^{2}\left[t_{2}^{2 p-2}-t_{1}^{2 p-2}\right]+2 \gamma\left[h\left(t_{2}\right)+h\left(t_{1}\right)\right]}{\left[1+\gamma^{2} t_{2}^{2 p-2}+2 \gamma h\left(t_{2}\right)\right]\left[1+\gamma^{2} t_{1}^{2 p-2}-2 \gamma h\left(t_{1}\right)\right]} .
$$

Since $r_{1}^{2}\left(t_{1}\right) \sim\left\{\begin{array}{ll}t_{1}^{2}\left(1+\gamma^{2}\right), & p=1, \\ t_{1}^{2}, & p<1,\end{array}\right.$ as $\quad r \rightarrow \infty$, and since $h(t) \rightarrow 0$ as $t \rightarrow \infty$,

$$
t_{1}^{2}-t_{2}^{2} \sim \begin{cases}\frac{2 t_{1}^{2} \gamma}{1+\gamma^{2}}\left[h\left(t_{2}\right)+h\left(t_{1}\right)\right], & p=1 \\ t_{1}^{2}\left[\gamma^{2}\left[t_{2}^{2 p-2}-t_{1}^{2 p-2}\right]+2 \gamma\left[h\left(t_{2}\right)+h\left(t_{1}\right)\right],\right. & p<1\end{cases}
$$

as $r \rightarrow \infty$.

If $p=1$, we get

$$
\frac{t_{1}^{2}-t_{2}^{2}}{t_{2}^{p-1} t_{1}^{2}} \rightarrow 0 \quad \text { as } \quad r \rightarrow \infty
$$

If $p<1$,

$\frac{t_{1}^{2}-t_{2}^{2}}{t_{2}^{p-1} t_{1}^{2}} \sim t_{2}^{p-1}\left[\gamma^{2}\left[1-\left(\frac{t_{1}}{t_{2}}\right)^{2 p-2}\right]+2 \gamma t_{2}^{2-2 p} h\left(t_{2}\right)+2 \gamma\left(\frac{t_{2}}{t_{1}}\right)^{2-2 p} t_{1}^{2-2 p} h\left(t_{1}\right)\right] \rightarrow 0$

as $r \rightarrow \infty$, using (3.7) and (2.9).

In any case, the hypotheses of Lemma 3.6 are fulfilled and the lemma yields

$$
\frac{f\left(t_{1}\right)-f\left(t_{2}\right)}{t_{2}^{p-1}} \rightarrow 0 \quad \text { as } \quad r \rightarrow \infty .
$$

For $p=1$, (2.4) $-(2.5)$ imply

$$
\begin{aligned}
& \theta_{1}\left(r_{1}^{-1}(r)\right)-\theta_{2}\left(r_{2}^{-1}(r)\right)=\theta_{1}\left(t_{1}\right)-\theta_{2}\left(t_{2}\right) \\
& \quad=f\left(t_{1}\right)-f\left(t_{2}\right)+\arccos \frac{1-\gamma h\left(t_{1}\right)}{\sqrt{1+\gamma^{2}-2 \gamma h\left(t_{1}\right)}}+\arccos \frac{1+\gamma h\left(t_{2}\right)}{\sqrt{1+\gamma^{2}+2 \gamma h\left(t_{2}\right)}} \\
& \quad \rightarrow 2 \arccos \frac{1}{\sqrt{1+\gamma^{2}}}
\end{aligned}
$$

(since $h(t) \rightarrow 0$ as $t \rightarrow \infty$ ).

For $p<1$, as $r \rightarrow \infty$

$$
\begin{aligned}
& \frac{\theta_{1}\left(r_{1}^{-1}(r)\right)-\theta_{2}\left(r_{2}^{-1}(r)\right)}{r^{p-1}}=\frac{\theta\left(t_{1}\right)-\theta\left(t_{2}\right)}{\left[r_{2}\left(t_{2}\right)\right]^{p-1}} \\
& \sim \frac{\theta\left(t_{1}\right)-\theta\left(t_{2}\right)}{t_{2}^{p-1}} \text { by (3.6) } \\
& =\frac{f\left(t_{1}\right)-f\left(t_{2}\right)}{t_{2}^{p-1}}+\left(\frac{t_{1}}{t_{2}}\right)^{p-1} \frac{1}{t_{1}^{p-1}} \arccos \frac{1-\gamma h\left(t_{1}\right)}{\sqrt{1+\gamma^{2} t_{1}^{2 p-2}-2 \gamma h\left(t_{1}\right)}} \\
& +\frac{1}{t_{2}^{p-1}} \arccos \frac{1+\gamma h\left(t_{2}\right)}{\sqrt{1+\gamma^{2} t_{2}^{2 p-2}+2 \gamma h\left(t_{2}\right)}} \\
& \rightarrow 0+\gamma+\gamma=2 \gamma
\end{aligned}
$$

by (3.8), (3.7) and Lemma 3.5 


\section{ON CONFORMAL MAPPINGS}

In this section we summarize some results on conformal mappings. The first theorem is taken from Theorem X on p. 315 of Warschawski (1942). Before stating the result, we establish some terminology.

Let $\mathcal{C}_{1}$ and $\mathcal{C}_{2}$ be two curves in the $w$-plane $(w=u+i v)$ given by continuous functions $v=\phi_{i}(u), u \geq u_{0}$, where $\phi_{1}(u)>\phi_{2}(u)$. Let $\mathcal{C}_{3}$ be a Jordan curve lying in the half-plane $u \leq u_{0}$, connecting the finite endpoints of $\mathcal{C}_{1}$ and $\mathcal{C}_{2}$. The curve $\mathcal{C}$ consisting of $\mathcal{C}_{1}, \mathcal{C}_{2}, \mathcal{C}_{3}$ divides the plane into 2 regions; denote by $E$ that part containing $\phi_{2}(u)<v<\phi_{1}(u), u>u_{0}$. We say $E$ is an $L$-strip with boundary inclination $\gamma$ at $u=\infty,|\gamma|<\frac{\pi}{2}$, if

$$
\lim _{\substack{u_{1}, u_{2} \rightarrow \infty \\ u_{2}>u_{1}}} \frac{\phi_{i}\left(u_{2}\right)-\phi_{i}\left(u_{1}\right)}{u_{2}-u_{1}}=\tan \gamma, \quad i=1,2 .
$$

Define

$$
\psi(u)=\frac{\phi_{1}(u)+\phi_{2}(u)}{2}, \quad \theta(u)=\phi_{1}(u)-\phi_{2}(u) .
$$

Let

$$
W_{\beta}=\{\tilde{z}=\tilde{x}+i \tilde{y}:|\tilde{y}|<\beta\},
$$

and suppose $J: E \rightarrow W_{\pi / 2}$ is a surjective conformal mapping such that $\lim _{u \rightarrow \infty} J(w)$ $=\infty$.

Theorem 4.1 (Warschawski). $\quad$ (i) For $w=u+i v \in E$, uniformly in $v$,

$$
\lim _{u \rightarrow \infty} \frac{J(w)}{\int_{u_{0}}^{u} \frac{d t}{\theta(t)}}=\pi
$$

(ii) For $\beta \in\left(0, \frac{\pi}{2}\right)$, set

$$
\begin{gathered}
T_{\beta}=J^{-1}\left(W_{\beta}\right), \\
S_{\beta}=\left\{w=u+i v: u>u_{0},\left|\frac{v-\psi(u)}{\theta(u)}\right|<\frac{\beta}{\pi}\right\} . \\
\text { If } \varepsilon>0 \text { and } 0<\beta \pm \varepsilon<\frac{\pi}{2}, \text { then there exists } N=N(\varepsilon, \beta) \text { such that } \\
S_{\beta-\varepsilon} \cap\{u \geq N\} \subseteq T_{\beta} \cap\{u \geq N\} \subseteq S_{\beta+\varepsilon} \cap\{u \geq N\} .
\end{gathered}
$$

(iii) For any $S_{\beta}, 0<\beta<\frac{\pi}{2}$,

$$
\lim _{u \rightarrow \infty}\left|J^{\prime}(w)\right| \theta(u)=\pi, \quad w=u+i v \in S_{\beta}, \text { uniformly on } v .
$$

We will make use of the following setup. Let $R$ be a domain in the $z$-plane and suppose $G$ is a conformal map of $R$ into the $\tilde{z}$-plane. If $Z_{t}$ is Brownian motion in the $z$-plane, then by conformal invariance, we can represent $G\left(Z_{t}\right)$ as a time-changed Brownian motion in the $\tilde{z}$-plane. Indeed, let $\widetilde{Z}(t)=\widetilde{Z}_{t}$ be Brownian motion in the $\tilde{z}$-plane started at $G\left(Z_{0}\right)$, and define

$$
\eta_{t}^{-1}=\int_{0}^{t}\left|G^{\prime}\left(G^{-1}\left(\widetilde{Z}_{s}\right)\right)\right|^{-2} d s
$$

Then

$$
G\left(Z_{t}\right)=_{\mathcal{L}} \widetilde{Z}\left(\eta_{t}\right)
$$

$(=\mathcal{L}$ means "have the same law"). 
For domains $R$ and $\widetilde{R}$ in the $z$-plane and the $\tilde{z}$-plane respectively, let

$$
\begin{aligned}
& \tau_{R}=\inf \left\{t>0: Z_{t} \notin R\right\}, \\
& \tilde{\tau}_{\widetilde{R}}=\inf \left\{t>0: \widetilde{Z}_{t} \notin \widetilde{R}\right\} .
\end{aligned}
$$

Then for $\tilde{z}=G(z)$,

$$
\begin{aligned}
P_{z}\left(\tau_{R}>t\right) & =P_{z}\left(Z_{s} \in R \forall s \leq t\right) \\
& =P_{z}\left(G\left(Z_{s}\right) \in G(R) \forall s \leq t\right) \\
& =P_{\tilde{z}}\left(\widetilde{Z}\left(\eta_{s}\right) \in G(R) \forall s \leq t\right) \\
& =P_{\tilde{z}}\left(\tilde{\tau}_{G(R)}>\eta_{t}\right) \\
& =P_{\tilde{z}}\left(\eta^{-1}\left(\tilde{\tau}_{G(R)}\right)>t\right) \\
& =P_{\tilde{z}}\left(\int_{0}^{\tilde{\tau}_{G(R)}}\left|G^{\prime}\left(G^{-1}\left(\widetilde{Z}_{s}\right)\right)\right|^{-2} d s>t\right) .
\end{aligned}
$$

\section{Functionals of Brownian motion in a STRIP}

Below we will show that our main theorems on exit times from twisted domains reduce to the following results. Recall $W_{\beta}=\{\tilde{z}=\tilde{x}+i \tilde{y}:|\tilde{y}|<\beta\}$.

Theorem 5.1. $\quad$ a) Suppose $\widetilde{D}$ is a bounded open subset of $\{\tilde{z}=\tilde{x}+i \tilde{y}:|\tilde{y}|<$ $\left.\frac{\pi}{2}\right\}$ with smooth boundary. Let $\beta \in\left(0, \frac{\pi}{2}\right)$ and suppose $\tilde{\tau}$ is the exit time of Brownian motion $\widetilde{Z}_{t}$ from

$$
\widetilde{W}_{\beta}=\widetilde{D} \cup\left(W_{\beta} \cap\{\tilde{x}>M\}\right) .
$$

Then for $p<1$ and $\tilde{z} \in \widetilde{W}_{\beta}$, with $\widetilde{Z}_{1}$ denoting the real part of $\widetilde{Z}$,

$$
-A_{1} \leq \liminf _{t \rightarrow \infty} t^{-\frac{1-p}{1+p}} \log P_{\tilde{z}}\left(\int_{0}^{\tilde{\tau}}\left[1+\left|\widetilde{Z}_{1}(s)\right|\right]^{\frac{2 p}{1-p}} d s>t\right),
$$

where

$$
A_{1}=\left[\pi^{1-p} 2^{2 p-1}(1-p)^{p}\right]^{-\frac{2}{1+p}} C_{p, 2 \beta / \pi}
$$

and $C_{p, \beta / 2 \pi}$ is from (1.3).

b) Moreover, for any $a>0$,

$$
-\frac{\pi}{2 a \beta} \leq \liminf _{t \rightarrow \infty}[\log t]^{-1} \log P_{\tilde{z}}\left(\int_{0}^{\tilde{\tau}} \exp \left(a \widetilde{Z}_{1}(s)\right) d s>t\right) .
$$

Theorem 5.2. $\quad$ a) Let $\tilde{\tau}$ be the exit time of Brownian motion $\widetilde{Z}_{t}$ from $W_{\beta} \cap$ $\{\tilde{x}>M\}, 0<\beta<\frac{\pi}{2}$. Then, for $p<1, \tilde{z} \in W_{\beta} \cap\{\tilde{x}>M\}$ and $A_{1}$ from Theorem 5.1 a),

$$
\limsup _{t \rightarrow \infty} t^{-\frac{1-p}{1+p}} \log P_{\tilde{z}}\left(\int_{0}^{\tilde{\tau}}\left[1+\left|\widetilde{Z}_{1}(s)\right|\right]^{\frac{2 p}{1-p}} d s>t\right) \leq-A_{1} .
$$

b) Moreover, for any $a>0$,

$$
\limsup _{t \rightarrow \infty}[\log t]^{-1} \log P_{\tilde{z}}\left(\int_{0}^{\tilde{\tau}} \exp \left(a \widetilde{Z}_{1}(s)\right) d s>t\right) \leq-\frac{\pi}{2 a \beta} .
$$


Before giving the proof, we describe the idea. Warschawski's theorem gives a conformal mapping $J$ of

$$
R=\left\{z=x+i y: x>0,|y|<x^{p}\right\}
$$

such that for $\tilde{z}=\tilde{x}+i \tilde{y}$,

$$
\left|J^{\prime}\left(J^{-1}(\tilde{z})\right)\right|^{-2} \approx[1+|\tilde{x}|]^{\frac{2 p}{1-p}} .
$$

Moreover, $J(R)$ is not too different from $W_{\beta} \cap\{\tilde{x}>M\}$, and so $\tilde{\tau}_{J(R)} \approx \tilde{\tau}$. Then by (4.5)

$$
P_{z}\left(\tau_{R}>t\right) \approx P_{\tilde{z}}\left(\int_{0}^{\tilde{\tau}}\left[1+\left|\widetilde{Z}_{1}(s)\right|\right]^{\frac{2 p}{1-p}} d s>t\right) .
$$

The asymptotics of the left hand side are known (Li, 2002), and the desired conclusion follows. The second part is a bit easier. The mapping $J(z)=\frac{2}{a} \log z$ takes

onto the strip $W_{\beta}$,

$$
R=\left\{z=r e^{i \theta}: r>0,-\frac{a \beta}{2}<\theta<\frac{a \beta}{2}\right\}
$$

$$
\left|J^{\prime}\left(J^{-1}(\tilde{z})\right)\right|^{-2} \approx e^{a \tilde{x}}
$$

and $\tau_{J(R)} \approx \tilde{\tau}$. By (4.5) we have

$$
P_{z}\left(\tau_{R}>t\right) \approx P_{\tilde{z}}\left(\int_{0}^{\tilde{\tau}} \exp \left(a \widetilde{Z}_{1}(s)\right) d s>t\right) .
$$

Again the asymptotics of the left hand side are known, and the desired conclusion follows.

First, we describe the asymptotics of $P_{z}\left(\tau_{R}>t\right)$ for $R$ as in the two cases above.

Lemma 5.3. For $R=R_{b}=\left\{x+i y:|y|<b x^{p}, x>0\right\}, b>0, p<1$, for any compact set $K \subseteq R$,

$$
\lim _{t \rightarrow \infty} t^{-\frac{1-p}{1+p}} \log P_{z}\left(\tau_{R}>t\right)=-C_{p, b}
$$

uniformly for $z \in K$, where $C_{p, b}$ is from (1.3).

Proof. Lifshits and Shi (2002) consider the domain

$$
\left\{(x, y): y>a|x|^{q}\right\}, \quad q>1,
$$

and compute the limit for $K=\{z\}$. In our setting their results translate into

$$
\lim _{t \rightarrow \infty} t^{-\frac{1-p}{1+p}} \log P_{z}\left(\tau_{R}>t\right)=-C_{p, b}
$$

for each $z \in R$. To extend this to the version we need, note that for each compact set $K \subseteq R$, there are a translation $\mathcal{R}_{1}$ of $R$ and $z_{1} \in \mathcal{R}_{1}$ with the following property: for any $z \in K$, there exists a translation $\widetilde{\mathcal{R}}_{1}$ of $\mathcal{R}_{1}$ where $z_{1} \in \mathcal{R}_{1}$ is shifted to $z \in \widetilde{\mathcal{R}}_{1}$ and $\widetilde{\mathcal{R}}_{1} \subseteq R$. Hence

$$
\begin{aligned}
P_{z}\left(\tau_{R}>t\right) & \geq P_{z}\left(\tau_{\tilde{\mathcal{R}}_{1}}>t\right) \\
& =P_{z_{1}}\left(\tau_{\mathcal{R}_{1}}>t\right) .
\end{aligned}
$$

The point is that $z_{1}$ and $\mathcal{R}_{1}$ are independent of $z \in K$. Similarly, there are a translation $\mathcal{R}_{2}$ of $R$ and $z_{2} \in \mathcal{R}_{2}$ such that

$$
P_{z}\left(\tau_{R}>t\right) \leq P_{z_{2}}\left(\tau_{\mathcal{R}_{2}}>t\right), \quad z \in K
$$


Combined with the previous inequality and the result of Lifshits and Shi, we get the desired uniform limiting behavior.

Lemma 5.4. For $R=R_{a, \beta}=\left\{r e^{i \theta}: r>0,-\frac{a \beta}{2}<\theta<\frac{a \beta}{2}\right\}, a>0,0<\beta<\pi / 2$, for any compact set $K \subseteq R$,

$$
\lim _{t \rightarrow \infty}(\log t)^{-1} \log P_{z}\left(\tau_{R}>t\right)=-\frac{\pi}{2 a \beta}, \text { uniformly for } z \in K,
$$

Proof. The region $R$ is a wedge with angle $a \beta$, and it is known that

$$
P_{z}\left(\tau_{R}>t\right) \sim C(z) t^{-\pi / 2 a \beta} \quad \text { as } t \rightarrow \infty
$$

(Spitzer, 1958). Thus

$$
\lim _{t \rightarrow \infty}[\log t]^{-1} \log P_{z}\left(\tau_{R}>t\right)=-\frac{\pi}{2 a \beta} .
$$

The rest of the proof is similar to that of Theorem 5.2 .

We need the following extension of Lemmas 5.3 and [5.4. Its proof is deferred to the end of this section.

Lemma 5.5. Let $R$ be $R_{b}$ or $R_{a, \beta}$ from Lemmas 5.3 or 5.4, and suppose $U$ is a bounded open set with smooth boundary such that $R \cap U \neq \emptyset$. Then for $z \in R \cup U$ and $C_{p, b}$ from (1.3),

$$
\begin{aligned}
& -C_{p, b} \leq \liminf _{t \rightarrow \infty} t^{-\frac{1-p}{1+p}} \log P_{z}\left(\tau_{R \cup U}>t\right), \quad R=R_{b}, \\
& -\frac{\pi}{2 a \beta} \leq \liminf _{t \rightarrow \infty}[\log t]^{-1} \log P_{z}\left(\tau_{R \cup U}>t\right), \quad R=R_{a, \beta} .
\end{aligned}
$$

Proof of Theorem 5.1 a). We are going to use the Warschawski mapping from the $z$-plane to the $\tilde{z}$-plane. Thus the variables from section four will be $x+i y$ instead of $u+i v$. Let

$$
R=\left\{x+i y:|y|<x^{p}, x>0\right\} .
$$

In Warschawski's theorem (Theorem 4.1) we use

$$
\begin{gathered}
\psi(x)=0, \\
\theta(x)=2 x^{p}, \\
x_{0}=0 .
\end{gathered}
$$

Thus, for the mapping $J: R \rightarrow W_{\pi / 2}$ described there,

$$
\begin{aligned}
& T_{\beta}=J^{-1}\left(W_{\beta}\right)=J^{-1}(\{\tilde{x}+i \tilde{y}:|\tilde{y}|<\beta\}), \\
& S_{\beta}=\left\{x+i y:|y|<\frac{2 \beta}{\pi} x^{p}, x>0\right\} .
\end{aligned}
$$

By Theorem 4.1, for $0<\varepsilon<\beta$ with $\beta+\varepsilon<\frac{\pi}{2}$ there exists $N=N(\varepsilon)$ such that

$$
S_{\beta-\varepsilon} \cap\{x \geq N\} \subseteq T_{\beta} \cap\{x \geq N\} \subseteq S_{\beta+\varepsilon} \cap\{x \geq N\} .
$$

Moreover,

$$
\lim _{x \rightarrow \infty}\left|J^{\prime}(z)\right| x^{p}=\frac{\pi}{2}, \quad z=x+i y \in S_{\beta+\varepsilon}, \text { uniformly in } y,
$$

and

$$
\lim _{x \rightarrow \infty} \frac{J(z)}{x^{1-p}}=2(1-p) \pi, \text { uniformly in } y .
$$


Together these give (recall that $\tilde{z}=J(z)$ )

$$
\left|J^{\prime}\left(J^{-1}(\tilde{z})\right)\right| \sim \frac{\pi}{2}[2(1-p)]^{\frac{p}{1-p}} \tilde{x}^{-\frac{p}{1-p}} \quad \text { as } \quad \tilde{x} \rightarrow \infty,
$$

and $\tilde{z}=\tilde{x}+i \tilde{y} \in J\left(S_{\beta+\varepsilon}\right)$, uniformly in $\tilde{y}$.

Hence for each $\delta>0$, for $M_{1}$ sufficiently large and $\tilde{z} \in J\left(S_{\beta+\varepsilon}\right)$,

$$
\begin{gathered}
(A-\delta)\left[[1+|\tilde{x}|]^{-\frac{p}{1-p}} I_{|\tilde{x}|>M_{1}}+K_{1} I_{|\tilde{x}| \leq M_{1}}\right] \leq\left|J^{\prime}\left(J^{-1}(\tilde{z})\right)\right| \\
\leq(A+\delta)\left[[1+|\tilde{x}|]^{-\frac{p}{1+p}} I_{|\tilde{x}|>M_{1}}+K_{2} I_{|\tilde{x}| \leq M_{1}}\right]
\end{gathered}
$$

where

$$
A=\frac{\pi}{2}[2(1-p)]^{\frac{p}{1-p}}=\pi\left[2^{2 p-1}(1-p)^{p}\right]^{\frac{1}{1-p}}
$$

and $K_{1}, K_{2}$ are unimportant constants depending on $M_{1}, \delta, p$.

Let $M$ be as in the hypothesis of the theorem. By (5.1), making $M_{1}$ larger if necessary, we can assume $M_{1}>M$ and

$$
W_{\beta} \cap\left\{\tilde{x} \geq M_{1}\right\}=J\left(T_{\beta}\right) \cap\left\{\tilde{x} \geq M_{1}\right\} \subseteq J\left(S_{\beta+\varepsilon} \cap\{x \geq N\}\right) .
$$

Then use (5.1) to choose $N_{1}>N$ such that

$$
J\left(S_{\beta-\varepsilon} \cap\left\{x \geq N_{1}\right\}\right) \subseteq J\left(T_{\beta}\right) \cap\left\{\tilde{x} \geq M_{1}\right\}=W_{\beta} \cap\left\{\tilde{x} \geq M_{1}\right\} .
$$

The translation $G_{1}$ of $S_{\beta-\varepsilon}$ defined by

$$
G_{1}=\left\{x+i y:|y|<\frac{2(\beta-\varepsilon)}{\pi}\left(x-N_{1}\right)^{p}, x>N_{1}\right\}
$$

satisfies

$$
G_{1} \subseteq S_{\beta-\varepsilon} \cap\left\{x \geq N_{1}\right\}
$$

Let $\tilde{z} \in \widetilde{W}_{\beta}=\widetilde{D} \cup\left(W_{\beta} \cap\{\tilde{x}>M\}\right)$ (from our hypothesis) and suppose $U \subseteq R$ is a bounded open set, with smooth boundary, such that $G_{1} \cap U \neq \emptyset$ and

$$
\tilde{z} \in J(U) \subseteq \widetilde{W}_{\beta}
$$

Then by (5.4)-5.5

$$
\begin{aligned}
\tilde{z} \in J\left(U \cup G_{1}\right) & \subseteq \widetilde{W}_{\beta} \cup\left[W_{\beta} \cap\left\{\tilde{x} \geq M_{1}\right\}\right) \\
& =\widetilde{W}_{\beta} .
\end{aligned}
$$

In particular, for $\tilde{\tau}_{1}=\tilde{\tau}\left(J\left(U \cup G_{1}\right)\right)=\inf \left\{t>0: \widetilde{Z}_{t} \notin J\left(U \cup G_{1}\right)\right\}$, we get $\tilde{\tau}_{1} \leq \tilde{\tau}$. For typographical simplicity, write

$$
F(z)=\left[1+\left|z_{1}\right|\right]^{\frac{2 p}{1-p}} .
$$


Then for $z=J^{-1}(\tilde{z})$,

$$
\begin{aligned}
P_{\tilde{z}}\left(\int_{0}^{\tilde{\tau}}\left[1+\left|\widetilde{Z}_{1}(s)\right|\right]^{\frac{2 p}{1-p}} d s>t\right)=P_{\tilde{z}}\left(\int_{0}^{\tilde{\tau}} F(\widetilde{Z}(s)) d s>t\right) \\
\quad \geq P_{\tilde{z}}\left(\int_{0}^{\tilde{\tau}_{1}} F(\widetilde{Z}(s)) d s>t\right) \\
\quad=P_{\tilde{z}}\left(\int_{0}^{\tilde{\tau}_{1}}\left[F(\widetilde{Z}(s)) I\left(\left|\widetilde{Z}_{1}(s)\right|>M_{1}\right)+K_{1}^{-2} I\left(\left|\widetilde{Z}_{1}(s)\right| \leq M_{1}\right)\right] d s\right. \\
\left.\quad+\int_{0}^{\tilde{\tau}_{1}} F(\widetilde{Z}(s)) I\left(\left|\widetilde{Z}_{1}(s)\right| \leq M_{1}\right) d s-\int_{0}^{\tilde{\tau}_{1}} K_{1}^{-2} I\left(\left|\widetilde{Z}_{1}(s)\right| \leq M_{1}\right) d s>t\right) \\
\quad \geq P_{\tilde{z}}\left(\int_{0}^{\tilde{\tau}_{1}}(A-\delta)^{2}\left|J^{\prime}\left(J^{-1}(\widetilde{Z}(s))\right)\right|^{-2} d s-\int_{0}^{\tilde{\tau}_{1}} K_{3} I\left(\left|\widetilde{Z}_{1}(s)\right| \leq M_{1}\right) d s>t\right)
\end{aligned}
$$

(by (5.2), where $K_{3}$ is independent of $t$ )

$$
\begin{aligned}
\geq & P_{\tilde{z}}\left(\int_{0}^{\tilde{\tau}_{1}}\left|J^{\prime}\left(J^{-1}(\widetilde{Z}(s))\right)\right|^{-2} d s>\left[(A-\delta)^{-2}+\delta\right] t\right) \\
& -P_{\tilde{z}}\left(\int_{0}^{\tilde{\tau}_{1}} I\left(\left|\widetilde{Z}_{1}(s)\right| \leq M_{1}\right) d s>K_{4} t\right)
\end{aligned}
$$

(where $K_{4}$ is independent of $t$ )

$$
=P_{z}\left(\tau\left(U \cup G_{1}\right)>\left[(A-\delta)^{-2}+\delta\right] t\right)-P_{\tilde{z}}\left(\int_{0}^{\tilde{\tau}_{1}} I\left(\left|\widetilde{Z}_{1}(s)\right| \leq M_{1}\right) d s>K_{4} t\right)
$$

(by (4.5)). By Lemma 5.5 and translation invariance of Brownian motion,

$\liminf _{t \rightarrow \infty} t^{-\frac{1-p}{1+p}} \log P_{z}\left(\tau\left(U \cup G_{1}\right)>\left[(A-\delta)^{-2}+\delta\right] t\right) \geq-\left[(A-\delta)^{-2}+\delta\right]^{\frac{1-p}{1+p}} C_{p, 2(\beta-\varepsilon) / \pi}$.

Since $J\left(U \cup G_{1}\right) \subseteq W_{\frac{\pi}{2}}, \tilde{\tau}_{1}$ is bounded by the first exit time $\eta$ of $\widetilde{Z}$ from $W_{\frac{\pi}{2}}$. It is well-known that for some $K_{5}>0$,

$$
\log P_{\tilde{z}}(\eta>t) \leq-K_{5} t, \quad t \text { large. }
$$

Thus, for $t$ large,

$$
\begin{aligned}
\log P_{\tilde{z}}\left(\int_{0}^{\tilde{\tau}_{1}} I\left(\left|\widetilde{Z}_{1}(s)\right| \leq M_{1}\right)>K_{4} t\right) & \leq \log P_{\tilde{z}}\left(\eta>K_{4} t\right) \\
& \leq-K_{5} t .
\end{aligned}
$$

Using this and (5.7), after taking logs on both sides of (5.6) we get

$$
\liminf _{t \rightarrow \infty} t^{-\frac{1-p}{1+p}} \log P_{\tilde{z}}\left(\int_{0}^{\tilde{\tau}}\left[1+\left|\widetilde{Z}_{1}(s)\right|\right]^{\frac{2 p}{1-p}} d s>t\right) \geq-\left[(A-\delta)^{-2}+\delta\right]^{\frac{1-p}{1+p}} C_{p, 2(\beta-\varepsilon) / \pi} .
$$


Let $\delta \rightarrow 0$ and then $\varepsilon \rightarrow 0$, to end up with

$$
\begin{aligned}
\liminf _{t \rightarrow \infty} t^{-\frac{1-p}{1+p}} \log P_{\tilde{z}}\left(\int_{0}^{\tilde{\tau}}\left[1+\left|\widetilde{Z}_{1}(s)\right|\right]^{\frac{2 p}{1+p}} d s>t\right) & \geq-A^{-\frac{2(1-p)}{1+p}} C_{p, 2 \beta / \pi} \\
& =-A_{1},
\end{aligned}
$$

as desired.

Proof of Theorem 5.2 a). We continue to use the notation from the proof of Theorem $5.1 \mathrm{a})$. Choose $\varepsilon>0$ so close to $\frac{\pi}{2}-\beta$ that

$$
W_{\beta} \cap\{\tilde{x}>M\} \subseteq J\left(S_{\beta+\varepsilon}\right) .
$$

This is possible because $S_{r} \uparrow R$ as $r \uparrow \pi / 2$. Then for $\tilde{z} \in W_{\beta} \cap\{\tilde{x}>M\}$ and $\tilde{\tau}_{2}=\inf \left\{t>0: \widetilde{Z}_{t} \notin J\left(S_{\beta+\varepsilon}\right)\right\}$, by (5.2),

$$
\begin{aligned}
P_{\tilde{z}}\left(\int_{0}^{\tilde{\tau}}\left[1+\left|\widetilde{Z}_{1}(s)\right|\right]^{\frac{2 p}{1-p}} d s>t\right) \leq P_{\tilde{z}}\left(\int_{0}^{\tilde{\tau}_{2}}\left[1+\left|\widetilde{Z}_{1}(s)\right|\right]^{\frac{2 p}{1-p}} d s>t\right) \\
\leq P_{\tilde{z}}\left(\int_{0}^{\tilde{\tau}_{2}}(A+\delta)^{2}\left|J^{\prime}\left(J^{-1}(\widetilde{Z}(s))\right)\right|^{-2} d s+K_{6} \int_{0}^{\tilde{\tau}_{2}} I\left(\left|\widetilde{Z}_{1}(s)\right| \leq M_{1}\right) d s>t\right) \\
\quad \leq P_{\tilde{z}}\left(\int_{0}^{\tilde{\tau}_{2}}\left|J^{\prime}\left(J^{-1}(\widetilde{Z}(s))\right)\right|^{-2} d s>(A+\delta)^{-2}(1-\delta) t\right) \\
\quad+P_{\tilde{z}}\left(\int_{0}^{\tilde{\tau}_{2}} I\left(\left|\widetilde{Z}_{1}(s)\right| \leq M_{1}\right) d s>K_{7} \delta t\right) \\
=P_{z}\left(\tau\left(S_{\beta+\varepsilon}\right)>(A+\delta)^{-2}(1-\delta) t\right) \\
\quad+P_{\tilde{z}}\left(\int_{0}^{\tilde{\tau}_{2}} I\left(\left|\widetilde{Z}_{1}(s)\right| \leq M_{1}\right) d s>K_{7} \delta t\right) .
\end{aligned}
$$

Since $S_{\beta+\varepsilon}=\left\{x+i y:|y|<\frac{2(\beta+\varepsilon)}{\pi} x^{p}, x>0\right\}$, we can use Lemma 5.3 on the first term. Now $J\left(S_{\beta+\varepsilon}\right) \subseteq W_{\pi / 2}$, and so $\tilde{\tau}_{2} \leq \eta$. Hence the log of the second term is bounded by $-K_{\delta} t$ for large $t$. Take the limsup as $t \rightarrow \infty$, then let $\delta \rightarrow 0, \varepsilon \rightarrow 0$, to end up with the upper bound

$$
\limsup _{n \rightarrow \infty} P_{\tilde{z}}\left(\int_{0}^{\tilde{\tau}}\left[1+\left|\widetilde{Z}_{1}(s)\right|\right]^{\frac{2 p}{1+p}} d s>t\right) \leq-A_{1} .
$$

Proof of Theorem $5.1 \mathrm{~b}$ ). Let

$$
J(z)=\frac{2}{a} \log z \quad \text { (principal branch) } .
$$

Given $\tilde{z} \in \widetilde{W}_{\beta}$, there exist a bounded open set $U$ with smooth boundary, and a translation $\mathcal{R}$ of

$$
R_{a, \beta}=\left\{r e^{i \theta}: r>0,-\frac{a \beta}{2}<\theta<\frac{a \beta}{2}\right\}
$$

such that

$$
\tilde{z} \in J(\mathcal{R} \cup U) \subseteq \widetilde{W}_{\beta}
$$


This is possible because $\widetilde{W}_{\beta} \subseteq\left\{\tilde{z}=\tilde{x}+i \tilde{y}\right.$ : $\left.|\tilde{y}|<\frac{\pi}{2}\right\}$. In particular, $\tilde{\tau} \geq \tilde{\tau}_{1}:=$ $\tilde{\tau}(J(\mathcal{R} \cup U))$. Since

$$
\left|J^{\prime}\left(J^{-1}(\tilde{z})\right)\right|=\frac{2}{a} e^{-a \tilde{x} / 2}, \quad \tilde{z}=\tilde{x}+i \tilde{y}
$$

we have

$$
\begin{aligned}
& P_{\tilde{z}}\left(\int_{0}^{\tilde{\tau}} \exp \left(a \widetilde{Z}_{1}(s)\right) d s>t\right)=P_{\tilde{z}}\left(\int_{0}^{\tilde{\tau}}\left|J^{\prime}\left(J^{-1}(\widetilde{Z}(s))\right)\right|^{-2} d s>\frac{a^{2} t}{4}\right) \\
& \quad \geq P_{\tilde{z}}\left(\int_{0}^{\tilde{\tau}_{1}}\left|J^{\prime}\left(J^{-1}(\widetilde{Z}(s))\right)\right|^{-2} d s>\frac{a^{2} t}{4}\right) \\
& \quad=P_{z}\left(\tau_{\mathcal{R} \cup U}>\frac{a^{2} t}{4}\right), z=J^{-1}(\tilde{z}) \quad(\text { by (4.5) }) .
\end{aligned}
$$

Together with translation invariance of Brownian motion and Lemma 5.5, this gives

$$
\liminf _{t \rightarrow \infty}[\log t]^{-1} \log P_{\tilde{z}}\left(\int_{0}^{\tilde{\tau}} \exp \left(a \widetilde{Z}_{1}(s)\right) d s>t\right) \geq-\frac{\pi}{2 a \beta},
$$

as desired.

Proof of Theorem 5.2 b). We continue to use the notation from the proof of Theorem $5.1 \mathrm{~b})$. Notice that $J$ maps the wedge

$$
R=R_{a, \beta}=\left\{r e^{i \theta}: r>0,-\frac{a \beta}{2}<\theta<\frac{a \beta}{2}\right\}
$$

onto $W_{\beta}$. Since $\tilde{\tau}=\tilde{\tau}\left(W_{\beta} \cap\{\tilde{x}>M\}\right) \leq \tilde{\tau}\left(W_{\beta}\right)$, we have

$$
\begin{aligned}
& P_{\tilde{z}}\left(\int_{0}^{\tilde{\tau}} \exp \left(a \widetilde{Z}_{1}(s)\right) d s>t\right) \leq P_{\tilde{z}}\left(\int_{0}^{\tilde{\tau}\left(W_{\beta}\right)} \exp \left(a \widetilde{Z}_{1}(s)\right) d s>t\right) \\
& \left.=P_{\tilde{z}}\left(\int_{0}^{\tilde{\tau}\left(W_{\beta}\right)}\left|J^{\prime}\left(J^{-1}(\widetilde{Z}(s))\right)\right|^{-2} d s>\frac{a^{2} t}{4}\right) \quad(\text { by (5.8) })\right) \\
& =P_{z}\left(\tau_{R}>\frac{a^{2} t}{4}\right), z=J^{-1}(\tilde{z}) \quad(\text { by (4.5) }) .
\end{aligned}
$$

Using Lemma 5.4 yields the desired conclusion.

We end this section with the proof of Lemma 5.5. As we will see, it comes down to the next lemma.

Lemma 5.6. Let $E \subseteq \mathbb{R}^{2}$ be a bounded open set with piecewise smooth boundary. Assume $I \subseteq \partial E$ is nonpolar for Brownian motion $Z_{t}$ and $z \in E, T>0$. Suppose $g(t)$ is twice differentiable with

$$
g(t) \uparrow \infty \quad \text { and } \quad g^{\prime}(t) \rightarrow 0 \quad \text { as } \quad t \rightarrow \infty
$$

and

$$
\int^{\infty} g^{\prime}(u) e^{\lambda u} d u=\infty \text { for all } \lambda>0
$$


Then there exists a positive constant $T_{1}$ such that

$$
\liminf _{t \rightarrow \infty}\left[g\left(t-T_{1}\right)\right]^{-1} \log \int_{T_{1}}^{t-T} e^{-g(t-s)} d_{s} P_{z}\left(Z\left(\tau_{E}\right) \in I, \tau_{E} \leq s\right) \geq-1 .
$$

Here $\tau_{E}=\inf \{t>0: Z(t) \notin E\}$.

Proof. For the harmonic function

$$
h(z)=P_{z}\left(Z\left(\tau_{E}\right) \in I\right)
$$

let $P_{z}^{h}$ denote the corresponding conditioned Brownian motion. Then

$$
\begin{aligned}
P_{z}\left(Z\left(\tau_{E}\right) \in I, \tau_{E} \leq s\right) & =P_{z}\left(Z\left(\tau_{E}\right) \in I\right)-P_{z}\left(Z\left(\tau_{E}\right) \in I, \tau_{E}>s\right) \\
& =P_{z}\left(Z\left(\tau_{E}\right) \in I\right)\left[1-P_{z}^{h}\left(\tau_{E}>s\right)\right] \\
& =h(z)\left[1-P_{z}^{h}\left(\tau_{E}>s\right)\right] .
\end{aligned}
$$

Consequently,

$$
d_{s} P_{z}\left(Z\left(\tau_{E}\right) \in I, \tau_{E} \leq s\right)=-h(z)\left[\frac{d}{d s} P_{z}^{h}\left(\tau_{E}>s\right)\right] d s .
$$

It is known (Bañuelos and Davis (1989)) that for some $C(z)$ and $\lambda>0$,

$$
P_{z}^{h}\left(\tau_{E}>t\right) \sim C(z) e^{-\lambda t} \quad \text { as } \quad t \rightarrow \infty .
$$

Given $\varepsilon>0$, choose $T_{1}>0$ such that

$$
(1-\delta) C(z) e^{-\lambda t} \leq P_{z}^{h}\left(\tau_{E}>t\right) \leq(1+\delta) C(z) e^{-\lambda t}, \quad t \geq T_{1} .
$$

Now for $t-T>T_{1}$,

$$
\begin{aligned}
{[h(z)]^{-1} } & \int_{T_{1}}^{t-T} e^{-g(t-s)} d_{s} P_{z}\left(Z\left(\tau_{E}\right) \in I, \tau_{E} \leq s\right) \\
= & -\int_{T_{1}}^{t-T} e^{-g(t-s)}\left[\frac{d}{d s} P_{z}^{h}\left(\tau_{E}>s\right)\right] d s \\
= & e^{-g\left(t-T_{1}\right)} P_{z}^{h}\left(\tau_{E}>T_{1}\right)-e^{-g(T)} P_{z}^{h}\left(\tau_{E}>t-T\right) \\
& +\int_{T_{1}}^{t-T} P_{z}^{h}\left(\tau_{E}>s\right) e^{-g(t-s)} g^{\prime}(t-s) d s .
\end{aligned}
$$

The boundary term can be written as

$$
e^{-g\left(t-T_{1}\right)}\left[P_{z}^{h}\left(\tau_{E}>T_{1}\right)-e^{-g(T)} P_{z}^{h}\left(\tau_{E}>t-T\right) e^{g\left(t-T_{1}\right)}\right] .
$$

Now

$$
\begin{aligned}
\int_{T_{1}}^{t-T} & P_{z}^{h}\left(\tau_{E}>s\right) e^{-g(t-s)} g^{\prime}(t-s) d s \\
\quad & (1-\delta) C(z) \int_{T_{1}}^{t-T} e^{-\lambda s-g(t-s)} g^{\prime}(t-s) d s(\text { by (5.10) }) \\
\quad= & (1-\delta) C(z) e^{-\lambda t} \int_{T}^{t-T_{1}} e^{\lambda u-g(u)} g^{\prime}(u) d u
\end{aligned}
$$


and

$$
\begin{aligned}
\lim _{t \rightarrow \infty} \frac{e^{-\lambda t} \int_{T}^{t-T_{1}} e^{\lambda u-g(u)} g^{\prime}(u) d u}{e^{-g\left(t-T_{1}\right)}} & =\lim _{t \rightarrow \infty} \frac{\int_{T}^{t-T_{1}} e^{\lambda u-g(u)} g^{\prime}(u) d u}{e^{\lambda t-g\left(t-T_{1}\right)}} \\
& =\lim _{t \rightarrow \infty} \frac{e^{\lambda\left(t-T_{1}\right)-g\left(t-T_{1}\right)} g^{\prime}\left(t-T_{1}\right)}{e^{\lambda t-g\left(t-T_{1}\right)}\left[\lambda-g^{\prime}\left(t-T_{1}\right)\right]} \\
& =0, \text { since } g^{\prime}(t) \rightarrow 0 \text { as } t \rightarrow \infty .
\end{aligned}
$$

Next observe that as $t \rightarrow \infty$

$$
\begin{aligned}
P_{z}^{h}\left(\tau_{E}>t-T\right) e^{g\left(t-T_{1}\right)} & \sim C(z) e^{-\lambda(t-T)+g\left(t-T_{1}\right)} \quad(\text { by }(5.8)) \\
& =C(z) \exp \left(-(t-T)\left[\lambda-\frac{g\left(t-T_{1}\right)}{t-T}\right]\right) \\
& \rightarrow 0,
\end{aligned}
$$

since $\frac{g\left(t-T_{1}\right)}{t-T} \rightarrow 0$ by L'Hôpital's rule and our hypotheses.

Thus, collecting all these relations,

$$
\begin{aligned}
& \liminf _{t \rightarrow \infty}\left[g\left(t-T_{1}\right)\right]^{-1} \log \int_{T_{1}}^{t-T} e^{-g(t-s)} d_{s} P_{z}\left(Z\left(\tau_{E}\right) \in I, \tau_{E} \leq s\right) \\
& =\liminf _{t \rightarrow \infty}\left[g\left(t-T_{1}\right)\right]^{-1}\left\{-g\left(t-T_{1}\right)+\log \left[P_{z}^{h}\left(\tau_{E}>T_{1}\right)\right.\right. \\
& -e^{-g(T)} P_{z}^{h}\left(\tau_{E}>t-T\right) e^{g\left(t-T_{1}\right)} \\
& \left.\left.\left.\quad+\frac{\int_{T_{1}}^{t-T} P_{z}^{h}\left(\tau_{E}>s\right) e^{-g(t-s)} g^{\prime}(t-s) d s}{e^{-g\left(t-T_{1}\right)}}\right]\right\} \quad \text { (by (5.9) }\right) \\
& \geq \liminf _{t \rightarrow \infty}\left[g\left(t-T_{1}\right)\right]^{-1}\left\{-g\left(t-T_{1}\right)+\log P_{z}^{h}\left(\tau_{E}>T_{1}\right)\right\} \quad(\text { by (5.12) } \\
& =-1,
\end{aligned}
$$

as desired.

Proof of Lemma 5.5. Let $z \in R \cup U$ and assume without loss of generality that $z \notin R$; otherwise we would have $P_{z}\left(\tau_{R \cup U}>t\right)>P_{z}\left(\tau_{R}>t\right)$, and the desired conclusions would follow from Lemmas 5.3 and 5.4 Let $z_{1} \in R \backslash \bar{U}$ and suppose $E$ is a bounded open set with smooth boundary such that $E \subseteq R \cup U, z \in E$ and $z_{1} \in \partial E$. Let $I \subseteq \partial E$ be a nonpolar set containing $z_{1}$.

Let $\delta>0$ and use Lemmas 5.3 and 5.4 to choose $T>0$ such that for $t>T$, uniformly for $w \in I$,

$$
\begin{aligned}
-\left(C_{p, b}+\delta\right) t^{\frac{1-p}{1+p}} & \leq \log P_{w}\left(\tau_{R}>t\right), & R & =R_{b}, \\
-\left(\frac{\pi}{2 a \beta}+\delta\right) \log t & \leq \log P_{w}\left(\tau_{R}>t\right), & & R=R_{a, \beta} .
\end{aligned}
$$

For typographical simplicity, write

$$
C= \begin{cases}C_{p, b}+\delta, & R=R_{b} \\ \frac{\pi}{2 a \beta}+\delta, & R=R_{a, \beta}\end{cases}
$$

and

$$
g(t)= \begin{cases}C t^{\frac{1-p}{1+p}}, & R=R_{b} \\ C \log t, & R=R_{a, \beta} .\end{cases}
$$


By the strong Markov property and (5.15)-(5.18),

$$
\begin{aligned}
P_{z}\left(\tau_{R \cup U}>t\right) & \geq P_{z}\left(t-\tau_{E}>T, Z\left(\tau_{E}\right) \in I, \tau_{R \cup U}>t\right) \\
& =\int_{0}^{t-T} \int P_{w}\left(\tau_{R \cup U}>t-s\right) P_{z}\left(\tau_{E} \in d s, Z\left(\tau_{E}\right) \in d w\right) \\
& \geq \int_{0}^{t-T} \int_{I} e^{-g(t-s)} P_{z}\left(\tau_{E} \in d s, Z\left(\tau_{E}\right) \in d w\right) \\
& =\int_{0}^{t-T} e^{-g(t-s)} d_{s} P_{z}\left(Z\left(\tau_{E}\right) \in I, \tau_{E} \leq s\right) .
\end{aligned}
$$

The function $g$ satisfies the hypothesis of Lemma 5.6, so for $T_{1}$ as there we have

$$
\begin{aligned}
& \liminf _{t \rightarrow \infty}\left[g\left(t-T_{1}\right)\right]^{-1} \log P_{z}\left(\tau_{R \cup U}>t\right) \\
& \quad \geq \liminf _{t \rightarrow \infty}\left[g\left(t-T_{1}\right)\right]^{-1} \int_{T_{1}}^{t-T} e^{-g(t-s)} d_{s} P_{z}\left(Z\left(\tau_{E}\right) \in I, \tau_{E} \leq s\right) \\
& \quad \geq-1 .
\end{aligned}
$$

The desired conclusions follow using (5.17)-(5.18) and then letting $\delta \rightarrow 0$.

\section{Conformal transformation of a neighborhood of $D$ to a strip}

The estimates for $J^{\prime}$ in Warschawski's theorem are not uniform for unrestricted $v$, and this causes some technical problems. We slightly enlarge the original domain $D$. Let $D_{\varepsilon} \supseteq \bar{D}$ be a twisted domain with growth radius $(\gamma+\varepsilon) r^{p}$. We assume $D$ and $D_{\varepsilon}$ have the same generating curve $f(r)$. Abusing the notation, let the boundary curves of $D_{\varepsilon}$ be given by

$$
\begin{aligned}
& C_{1}: \theta=f_{1}(r), \quad r \geq r_{1}, \\
& C_{2}: \theta=f_{2}(r), \quad r \geq r_{1}, \\
& C_{3}: \quad r=r_{1}, \quad f_{2}\left(r_{1}\right) \leq \theta \leq f_{1}\left(r_{1}\right) .
\end{aligned}
$$

By (2.11) we have (for $r_{0}$ large enough)

$$
f_{i}(r)=\theta_{i}\left(r_{i}^{-1}(r)\right), \quad r \geq r_{0},
$$

where $\theta_{i}$ and $r_{i}$ are from 2.2 -2.5) with $\gamma$ there replaced by $\gamma+\varepsilon$. Define

$$
E_{\varepsilon}=\left\{w=u+i v: u>\ln r_{1}, f_{2}\left(e^{u}\right)<v<f_{1}\left(e^{u}\right)\right\} .
$$

Lemma 6.1. The analytic mapping $F: E_{\varepsilon} \rightarrow D_{\varepsilon}$ given by $F(w)=e^{w}$ is one-toone and onto.

Proof. Assume $w_{j}=u_{j}+i v_{j} \in E_{\varepsilon}, j=1,2$, with $e^{w_{1}}=e^{w_{2}}$. Then $e^{u_{1}}=e^{u_{2}}$, yielding $u_{1}=u_{2}$. Thus for some integer $n, v_{1}=v_{2}+2 \pi n$. It is no loss to assume $n \geq 0$. Now

$$
\begin{aligned}
2 \pi n=v_{1}-v_{2} & <f_{1}\left(e^{u_{1}}\right)-f_{2}\left(e^{u_{2}}\right) \\
& =f_{1}\left(e^{u_{1}}\right)-f_{2}\left(e^{u_{1}}\right) \\
& <2 \pi
\end{aligned}
$$

by our hypothesis that the boundary curves given by $f_{1}$ and $f_{2}$ do not cross. Hence $n=0$, and consequently $v_{1}=v_{2}$. 
For surjectivity, let $x+i y \in D_{\varepsilon}$. With $(r, \theta)$ being the corresponding polar coordinates, let $w=\ln r+i \theta$. We know $r>r_{1}$ and $f_{2}(r)<\theta<f_{2}(r)$. Thus $w \in E_{\varepsilon}$ and $e^{w}=x+i y$.

The next step is to apply Warschawski's theorem to $E_{\varepsilon}$. First we verify that $E_{\varepsilon}$ is an $L$-strip with boundary inclination 0 at $u=\infty$; that is, we check (4.1) in the present context:

$$
\lim _{\substack{u_{1}, u_{2} \rightarrow \infty \\ u_{2}>u_{1}}} \frac{f_{i}\left(e^{u_{2}}\right)-f_{i}\left(e^{u_{1}}\right)}{u_{2}-u_{1}}=0, \quad i=1,2 .
$$

Indeed, for some $\tilde{u} \in\left(u_{1}, u_{2}\right)$,

$$
\begin{aligned}
\frac{f_{i}\left(e^{u_{2}}\right)-f_{i}\left(e^{u_{1}}\right)}{u_{2}-u_{1}} & \left.=\frac{\theta_{i}\left(r_{i}^{-1}\left(e^{u_{2}}\right)\right)-\theta_{i}\left(r_{i}^{-1}\left(e^{u_{1}}\right)\right)}{u_{2}-u_{1}} \quad \text { by (6.1) }\right) \\
& =\frac{\theta_{i}^{\prime}\left(r_{i}^{-1}\left(e^{\tilde{u}}\right)\right) e^{\tilde{u}}}{r_{i}^{\prime}\left(r_{i}^{-1}\left(e^{\tilde{u}}\right)\right)} \\
& =\theta_{i}^{\prime}\left(r_{i}^{-1}\left(e^{\tilde{u}}\right)\right) r_{i}^{-1}\left(e^{\tilde{u}}\right) \frac{e^{\tilde{u}}}{r_{i}^{-1}\left(e^{\tilde{u}}\right) r_{i}^{\prime}\left(r_{i}^{-1}\left(e^{\tilde{u}}\right)\right)}
\end{aligned}
$$

Since $\tilde{u} \rightarrow \infty$ as $u_{1}, u_{2} \rightarrow \infty$, by Lemma 3.3 and Corollary 2.2 the right hand side of (6.3) converges to 0 . Thus $E_{\varepsilon}$ is an $L$-strip with boundary inclination 0 at $u=\infty$, as claimed.

Let $J: E_{\varepsilon} \rightarrow W_{\pi / 2}=\left\{\tilde{z}=\tilde{x}+i \tilde{y}:|\tilde{y}|<\frac{\pi}{2}\right\}$ be the conformal mapping in Warschawski's theorem. Then

$$
\begin{aligned}
\psi(u) & =\frac{1}{2}\left[f_{2}\left(e^{u}\right)+f_{1}\left(e^{u}\right)\right], \\
\theta(u) & =f_{1}\left(e^{u}\right)-f_{2}\left(e^{u}\right) .
\end{aligned}
$$

Lemma 6.2. As $u \rightarrow \infty$,

$$
\theta(u) \sim \begin{cases}2(\gamma+\varepsilon) e^{(p-1) u}, & p<1, \\ 2 \arccos \frac{1}{\sqrt{1+(\gamma+\varepsilon)^{2}}}, & p=1 .\end{cases}
$$

Proof. By (6.1),

$$
\theta(u)=\theta_{1}\left(r_{1}^{-1}\left(e^{u}\right)\right)-\theta_{2}\left(r_{2}^{-1}\left(e^{u}\right)\right) \sim \begin{cases}2(\gamma+\varepsilon) e^{(p-1) u}, & p<1, \\ 2 \arccos \frac{1}{\sqrt{1+(\gamma+\varepsilon)^{2}}}, & p=1,\end{cases}
$$

by Theorem 3.1 (recall that our growth radius is $(\gamma+\varepsilon) r^{p}$, NOT $\left.\gamma r^{p}\right)$.

Define

$$
G=J \circ F^{-1}: D_{\varepsilon} \rightarrow W_{\pi / 2} .
$$

This is a conformal mapping from the enlarged twisted domain onto the strip $W_{\pi / 2}$. Recall that $S_{\beta}=\left\{w=u+i v: u>\ln r_{1},\left|\frac{v-\psi(u)}{\theta(u)}\right|<\frac{\beta}{\pi}\right\}$.

Lemma 6.3. a) Given $0<\beta<\frac{\pi}{2}, p<1$ and small $\delta>0$, for large $M$,

$$
\begin{aligned}
& \left(A_{p, \gamma+\varepsilon}-\delta\right)\left([1+|\tilde{x}|]^{-\frac{p}{1-p}} I_{|\tilde{x}|>M}+K_{1} I_{\mid \tilde{x} \leq M}\right) \leq\left|G^{\prime}\left(G^{-1}(\tilde{z})\right)\right| \\
& \quad \leq\left(A_{p, \gamma+\varepsilon}+\delta\right)\left([1+|\tilde{x}|]^{-\frac{p}{1-p}} I_{|\tilde{x}|>M}+K_{2} I_{|\tilde{x}| \leq M}\right),
\end{aligned}
$$


$\tilde{z}=\tilde{x}+i \tilde{y} \in J\left(S_{\beta}\right)$, where

$$
A_{p, \gamma}=\left[\frac{\pi^{p}}{2 \gamma(1-p)^{p}}\right]^{\frac{1}{1-p}}
$$

and $K_{1}, K_{2}$ are independent of $M$.

b) For $0<\beta<\frac{\pi}{2}, p=1, M \in \mathbb{R}$ and $\delta>0$ small, there are positive constants $C_{3}=C_{3}(\delta)$ and $C_{4}=C_{4}(\delta)$ such that

$C_{3} \exp \left(-C_{5}(1+\delta) \tilde{x}\right) \leq\left|G^{\prime}\left(G^{-1}(\tilde{z})\right)\right| \leq C_{4} \exp \left(-C_{5}(1-\delta) \tilde{x}\right), \quad \tilde{z} \in J\left(S_{\beta}\right), \tilde{x} \geq M$, where $C_{5}=\frac{2}{\pi} \arccos \left(\frac{1}{\sqrt{1+(\gamma+\varepsilon)^{2}}}\right)$.

Proof. By (6.5), writing $\tilde{z}=G(z)$ and $F^{-1}(z)=w=u+i v$,

$$
\begin{aligned}
\left.\mid G^{\prime}\left(G^{-1}(\tilde{z})\right)\right) \mid & =\left|G^{\prime}(z)\right| \\
& =\left|\frac{J^{\prime}\left(F^{-1}(z)\right)}{F^{\prime}\left(F^{-1}(z)\right)}\right| \\
& =\left|\frac{J^{\prime}(w)}{F^{\prime}(w)}\right| \\
& =\frac{\left|J^{\prime}(w)\right|}{e^{u}} \\
& \sim \frac{\pi}{\theta(u)} e^{-u} \quad \text { as } u \rightarrow \infty, w \in S_{\beta}, \text { uniformly in } v,
\end{aligned}
$$

by Theorem 4.1 (ii).

a) If $p<1$, then by Lemma 6.2, (6.6) yields

$$
\left|G^{\prime}\left(G^{-1}(\tilde{z})\right)\right| \sim \frac{1}{2(\gamma+\varepsilon)} e^{-p u} \text { as } u \rightarrow \infty, w \in S_{\beta} \text {, uniformly in } v .
$$

By Theorem 4.1 i) and Lemma 6.2 as $u \rightarrow \infty$, uniformly in $v$,

$$
\begin{aligned}
J(w) & \sim \pi \int_{\ln r_{1}}^{u} \frac{d t}{\theta(t)} \\
& \sim \frac{\pi}{2(\gamma+\varepsilon)(1-p)} e^{(1-p) u} .
\end{aligned}
$$

Since $\tilde{z}=G(z)=J\left(F^{-1}(z)\right)=J(w)$, this yields

$$
\tilde{x} \sim \frac{\pi}{2(\gamma+\varepsilon)(1-p)} e^{(1-p) u} \text { as } u \rightarrow \infty \text {, uniformly in } v .
$$

Combining this with (6.7), we get

$$
\left|G^{\prime}\left(G^{-1}(\tilde{z})\right)\right| \sim A_{p, \gamma+\varepsilon} \tilde{x}^{-\frac{p}{1-p}} \text { as } \tilde{x} \rightarrow \infty, \tilde{z}=\tilde{x}+i \tilde{y} \in J\left(S_{\beta}\right),
$$

uniformly in $\tilde{y}$. Part a) follows from this.

b) If $p=1$, then by Lemma 6.2, (6.6) implies

$$
\left|G^{\prime}\left(G^{-1}(\tilde{z})\right)\right| \sim C_{5}^{-1} e^{-u} \text { as } u \rightarrow \infty, w \in S_{\beta} \text {, uniformly in } v \text {. }
$$

By Theorem 4.1 i) and Lemma 6.2 as $u \rightarrow \infty$, uniformly in $v$,

$$
\tilde{z}=J(w) \sim C_{5}^{-1} u
$$

yielding

$$
\tilde{x} \sim C_{5}^{-1} u \text { as } u \rightarrow \infty \text { uniformly in } v .
$$


Let $\delta>0$ be small. For large $u$, by (6.9)

$$
(1-\delta) \tilde{x} \leq C_{5}^{-1} u \leq(1+\delta) \tilde{x},
$$

and so

$$
\exp \left(-C_{5}(1+\delta) \tilde{x}\right) \leq e^{-u} \leq \exp \left(-C_{5}(1-\delta) \tilde{x}\right) .
$$

Combined with (6.8), as $u \rightarrow \infty, w \in S_{\beta}$, uniformly in $v$, this yields

$$
C_{6} \exp \left(-C_{5}(1+\delta) \tilde{x}\right) \leq\left|G^{\prime}\left(G^{-1}(\tilde{z})\right)\right| \leq C_{7} \exp \left(-C_{5}(1+\delta) \tilde{x}\right) .
$$

The desired conclusion follows from this.

Lemma 6.4. For $\varepsilon>0$ small, there exist positive $\varepsilon_{1}, \varepsilon_{2}, N$ with $1>\varepsilon_{1}>\varepsilon_{2}$ such that for $\alpha_{i}=\frac{\pi}{2}\left(1-\varepsilon_{i}\right)$,

$$
S_{\alpha_{1}} \cap\{u \geq N\} \subseteq F^{-1}(D) \cap\{u \geq N\} \subseteq S_{\alpha_{2}} \cap\{u \geq N\} .
$$

We defer the proof to section 8.

Let $\alpha_{1}, \alpha_{2}, N$ be as in Lemma 6.4. By Theorem 4.1] for $\delta_{1}, \delta_{2}$ small and positive, there exists $\widetilde{N} \geq N$ such that

$$
\begin{aligned}
T_{\alpha_{1}-\delta_{1}} \cap\{u \geq \widetilde{N}\} & \subseteq S_{\alpha_{1}} \cap\{u \geq \widetilde{N}\} \quad \text { (by Theorem 4.1) } \\
& \subseteq F^{-1}(D) \cap\{u \geq \widetilde{N}\} \quad \text { (by Lemma 6.4) } \\
& \subseteq S_{\alpha_{2}} \cap\{u \geq \widetilde{N}\} \quad \text { (by Lemma 6.4) } \\
& \subseteq T_{\alpha_{2}+\delta_{2}} \cap\{u \geq \widetilde{N}\} \quad \text { (by Theorem 4.1) }
\end{aligned}
$$

This implies we can choose $M_{1}$ and $M_{2}$ such that

$$
W_{\alpha_{1}-\delta_{1}} \cap\left\{\tilde{x} \geq M_{2}\right\} \subseteq G(D) \cap\left\{\tilde{x} \geq M_{2}\right\} \subseteq W_{\alpha_{2}+\delta_{2}} \cap\left\{\tilde{x} \geq M_{1}\right\} .
$$

By making $\delta_{2}$ a bit larger and $M_{1}$ smaller, if necessary, we can take

$$
G(D) \subseteq W_{\alpha_{2}+\delta_{2}} \cap\left\{\tilde{x} \geq M_{1}\right\} .
$$

This is possible because $\bar{D} \subseteq D_{\varepsilon}$ and (6.11) holds. Similarly, by (6.10) and Theorem 4.1 we can choose $\beta \in\left(0, \frac{\pi}{2}\right)$ such that

$$
W_{\alpha_{2}+\delta_{2}} \cap\left\{\tilde{x} \geq M_{1}\right\} \subseteq J\left(S_{\beta}\right) .
$$

Remark 6.5. We need $\beta$ only for the case $p=1$. Notice too that (6.13) is true if $\beta$ is made larger.

\section{Proof of the Main Results}

We use the notation of $\S 6$.

Proof of Theorem 1.1. Let $z \in D$ and suppose $p<1$. Given $\delta>0$, making $M_{2}$ from (6.11) larger if necessary, by Lemma 6.3 a) we get

$$
\begin{aligned}
& \left(A_{p, \gamma+\varepsilon}-\delta\right)\left[[1+|\tilde{x}|]^{-\frac{p}{1-p}} I_{|\tilde{x}|>M_{2}}+K_{1} I_{|\tilde{x}| \leq M_{2}}\right] \leq\left|G^{\prime}\left(G^{-1}(\tilde{z})\right)\right| \\
& \quad \leq\left(A_{p, \gamma+\varepsilon}+\delta\right)\left[[1+|\tilde{x}|]^{-\frac{p}{1-p}} I_{|\tilde{x}|>M_{2}}+K_{2} I_{|\tilde{x}| \leq M_{2}}\right] .
\end{aligned}
$$


Here and in what follows, $K_{i}$ will denote a constant independent of $t$. By (6.11) there is a bounded open subset $\widetilde{D}$ of $W_{\pi / 2}$ such that $z \in \widetilde{D}$ and

$$
G(D) \supseteq\left[W_{\alpha_{1}-\delta_{1}} \cap\left\{\tilde{x}>M_{2}\right\}\right] \cup \widetilde{D} .
$$

Then, with $\eta=\tilde{\tau}\left(\widetilde{D} \cup\left[W_{\alpha_{1}-\delta_{1}} \cap\left\{\tilde{x}>M_{2}\right\}\right]\right)$,

$$
\begin{aligned}
P_{z}\left(\tau_{D}>t\right) & =P_{\tilde{z}}\left(\int_{0}^{\tilde{\tau}(G(D))}\left|G^{\prime}\left(G^{-1}(\widetilde{Z}(s))\right)\right|^{-2} d s>t\right)(\text { by }(4.5)) \\
\geq & P_{\tilde{z}}\left(\int_{0}^{\eta}\left|G^{\prime}\left(G^{-1}(\widetilde{Z}(s))\right)\right|^{-2} d s>t\right) \\
\geq & P_{\tilde{z}}\left(\int_{0}^{\eta}\left[1+\left|\widetilde{Z}_{1}(s)\right|\right]^{\frac{2 p}{1-p}} d s-K_{3} \int_{0}^{\eta} I\left(\left|\widetilde{Z}_{1}(s)\right| \leq M_{2}\right) d s>\left(A_{p, \gamma+\varepsilon}+\delta\right)^{2} t\right)
\end{aligned}
$$

(by (7.1))

$$
\begin{aligned}
& \geq P_{\tilde{z}}\left(\int_{0}^{\eta}\left[1+\left|\widetilde{Z}_{1}(s)\right|\right]^{\frac{2 p}{1-p}} d s>\left(A_{p, \gamma+\varepsilon}+2 \delta\right)^{2} t\right) \\
& -P_{\tilde{z}}\left(\int_{0}^{\eta} I\left(\left|\widetilde{Z}_{1}(s)\right| \leq M_{2}\right) d s>K_{4} t\right) .
\end{aligned}
$$

Now by (7.2) and (6.12), $\eta \leq \tilde{\tau}\left(W_{\pi / 2}\right)$, and it is well-known that

$$
\log P_{\tilde{z}}\left(\tilde{\tau}\left(W_{\pi / 2}\right)>t\right) \leq-K_{5} t, \quad t \text { large. }
$$

In particular, for $t$ large,

$$
\begin{aligned}
& \log P_{\tilde{z}}\left(\int_{0}^{\eta} I\left(\left|\widetilde{Z}_{1}(s)\right| \leq M_{2}\right) d s>K_{4} t\right) \\
& \quad \leq \log P_{\tilde{z}}\left(\tilde{\tau}\left(W_{\pi / 2}\right)>K_{4} t\right) \\
& \quad \leq-K_{6} t .
\end{aligned}
$$

Then by Theorem $5.1 \mathrm{a}$ ), (7.3) yields

$$
\liminf _{t \rightarrow \infty} t^{-\frac{1-p}{1+p}} \log P_{\tilde{z}}\left(\tau_{D}>t\right) \geq-B_{p} C_{p, 2\left(\alpha_{1}-\delta_{1}\right) / \pi}\left[A_{p, \gamma+\varepsilon}+2 \delta\right]^{\frac{2(1-p)}{1+p}},
$$

where

$$
B_{p}=\left[\pi^{1-p} 2^{2 p-1}(1-p)^{p}\right]^{-\frac{2}{1+p}} .
$$

As for the lim sup behavior, observe by (6.12) that

$$
\tilde{\tau}(G(D)) \leq \tau_{1}:=\tilde{\tau}\left(W_{\alpha_{2}+\delta_{2}} \cap\left\{\tilde{x}>M_{1}\right\}\right),
$$


and so

$$
\begin{aligned}
P_{z}\left(\tau_{D}>t\right)= & \left.P_{\tilde{z}}\left(\int_{0}^{\tilde{\tau}(G(D))}\left|G^{\prime}\left(G^{-1}(\widetilde{Z}(s))\right)\right|^{-2} d t>t\right) \quad \text { (by (4.5) }\right) \\
\leq & P_{\tilde{z}}\left(\int_{0}^{\tilde{\tau}_{1}}\left|G^{\prime}\left(G^{-1}(\widetilde{Z}(s))\right)\right|^{-2} d t>t\right) \\
\leq & P_{\tilde{z}}\left(\int_{0}^{\tau_{1}}\left[1+\left|\widetilde{Z}_{1}(s)\right|\right]^{\frac{2 p}{1-p}} d s\right. \\
& \left.\left.\quad+K_{7} \int_{0}^{\tau_{1}} I\left(\left|\widetilde{Z}_{1}(s)\right| \leq M_{2}\right) d s>\left(A_{p, \gamma+\varepsilon}-\delta\right)^{2} t\right) \quad \text { by (7.1) }\right) \\
\leq & P_{\tilde{z}}\left(\int_{0}^{\tau_{1}}\left[1+\left|\widetilde{Z}_{1}(s)\right|\right]^{\frac{2 p}{1-p}} d s>(1-\delta)\left(A_{p, \gamma+\varepsilon}-\delta\right)^{2} t\right) \\
& +P_{\tilde{z}}\left(K_{7} \int_{0}^{\tau_{1}} I\left(\left|\widetilde{Z}_{1}(s)\right| \leq M_{2}\right) d s>\delta\left(A_{p, \gamma+\varepsilon}-\delta\right)^{2} t\right) .
\end{aligned}
$$

Since $\tau_{1} \leq \tilde{\tau}\left(W_{\pi / 2}\right)$, the log of the second term on the left is bounded by

$$
\begin{aligned}
\log P_{\tilde{z}}\left(\tau_{1}>K_{8} t\right) & \leq \log P_{\tilde{z}}\left(\tilde{\tau}\left(W_{\pi / 2}\right)>K_{8} t\right) \\
& \leq-K_{9} t, \quad t \text { large. }
\end{aligned}
$$

Then, by Theorem 5.2 a), (7.7) yields

$$
\limsup _{t \rightarrow \infty} t^{-\frac{1-p}{1+p}} \log P_{z}\left(\tau_{D}>t\right) \geq-B_{p} C_{p, 2\left(\alpha_{2}+\delta_{2}\right) / \pi}\left[(1-\delta)\left(A_{p, \gamma+\varepsilon}-\delta\right)^{2}\right]^{\frac{1-p}{1+p}}
$$

where $B_{p}$ is from (7.5).

To finish, in the following order, let $\delta \rightarrow 0, \delta_{1} \rightarrow 0, \delta_{2} \rightarrow 0, \varepsilon_{1} \rightarrow 0, \varepsilon_{2} \rightarrow 0$ (which imply $\alpha_{i} \rightarrow \frac{\pi}{2}$ ) and $\varepsilon \rightarrow 0$ in (7.4) and (7.8) to get

$$
\lim _{t \rightarrow \infty} t^{-\frac{1-p}{1+p}} \log P_{z}\left(\tau_{D}>t\right)=B_{p} C_{p, 1}\left[A_{p, \gamma}\right]^{\frac{2(1-p)}{1+p}}
$$

as desired.

Proof of Theorem 1.2. Let $z \in D$. Suppose $p=1, \beta$ is from (6.13) and $M_{1}$ is from (6.12). Let $\delta>0$ be small and use Lemma 6.3 b) to choose positive $C_{3}(\delta), C_{4}(\delta)$ such that

$$
C_{3}(\delta) \exp \left(-C_{5}(1+\delta) \tilde{x}\right) \leq\left|G^{\prime}\left(G^{-1}(\tilde{z})\right)\right| \leq C_{4}(\delta) \exp \left(-C_{5}(1-\delta) \tilde{x}\right),
$$

for $\tilde{z}=\tilde{x}+i \tilde{y} \in J\left(S_{\beta}\right), \tilde{x} \geq M_{1}$, where

$$
C_{5}=\frac{2}{\pi} \arccos \frac{1}{\sqrt{1+(\gamma+\varepsilon)^{2}}}
$$

Exactly as in the preceding proof, there is a bounded open subset $\widetilde{D}$ of $W_{\pi / 2}$ such that $z \in \widetilde{D}$ and (7.2) holds. Then for $\eta=\tilde{\tau}\left(\widetilde{D} \cup\left[W_{\alpha_{1}-\delta_{1}} \cap\left\{\tilde{x}>M_{2}\right\}\right]\right)$ and $\tau_{1}=\tilde{\tau}\left(W_{\alpha_{2}+\delta_{2}} \cap\left\{\tilde{x}>M_{1}\right\}\right)$, by (4.5)

$$
\begin{aligned}
& P_{\tilde{z}}\left(\int_{0}^{\eta}\left|G^{\prime}\left(G^{-1}(\widetilde{Z}(s))\right)\right|^{-2} d s>t\right) \\
& \quad \leq P_{z}\left(\tau_{D}>t\right) \leq P_{\tilde{z}}\left(\int_{0}^{\tau_{1}}\left|G^{\prime}\left(G^{-1}(\widetilde{Z}(s))\right)\right|^{-2} d s>t\right) .
\end{aligned}
$$


Using (7.9),

$$
\begin{aligned}
& P_{\tilde{z}}\left(\int_{0}^{\eta} \exp \left(2 C_{5}(1-\delta) \widetilde{Z}_{1}(s)\right) d s>C_{4}^{2}(\delta) t\right) \leq P_{z}\left(\tau_{D}>t\right) \\
& \quad \leq P_{\tilde{z}}\left(\int_{0}^{\tilde{\tau}} \exp \left(2 C_{5}(1+\delta) \widetilde{Z}_{1}(s)\right) d s>C_{3}^{2}(\delta) t\right) .
\end{aligned}
$$

By Theorems $5.1 \mathrm{~b}$ ) and $5.2 \mathrm{~b}$ ),

$$
\begin{aligned}
-\frac{\pi}{4 C_{5}(1-\delta) \beta} & \leq \liminf _{t \rightarrow \infty}[\log t]^{-1} \log P_{z}\left(\tau_{D}>t\right) \\
& \leq \limsup _{t \rightarrow \infty}[\log t]^{-1} \log P_{z}\left(\tau_{D}>t\right) \\
& \leq-\frac{\pi}{4 C_{5}(1+\delta) \beta} .
\end{aligned}
$$

To finish, let $\delta \rightarrow 0$, then $\beta \rightarrow \frac{\pi}{2}$ (recall Remark 6.5) and then $\varepsilon \rightarrow 0$ to get

$$
\lim _{t \rightarrow \infty}[\log t]^{-1} \log P_{z}\left(\tau_{D}>t\right)=\pi\left[4 \arccos \frac{1}{\sqrt{1+\gamma^{2}}}\right]^{-1},
$$

as desired.

\section{Proof of Lemma 6.4}

We need to refer to the boundary curves of $D_{\varepsilon}$ and $D$. To distinguish them, we use tildes over the quantities associated with $D_{\varepsilon}$. In particular, the boundary curves of $D_{\varepsilon}$ will be

$$
\begin{aligned}
& \widetilde{C}_{i}: \quad \theta=\tilde{f}_{i}(r), \quad r \geq \tilde{r}_{1}, \quad i=1,2, \\
& \widetilde{C}_{3}: \quad r=\tilde{r}_{1}, \quad \tilde{f}_{2}\left(\tilde{r}_{1}\right) \leq \theta \leq \tilde{f}_{2}\left(\tilde{r}_{1}\right) .
\end{aligned}
$$

By (2.11), for $r_{0}$ large and for $i=1,2$,

$$
\begin{aligned}
& f_{i}(r)=\theta_{i}\left(r_{i}^{-1}(r)\right), \quad r \geq r_{0}, \\
& \tilde{f}_{i}(r)=\tilde{\theta}_{i}\left(\tilde{r}_{i}^{-1}(r)\right), \quad r \geq r_{0},
\end{aligned}
$$

where $\theta_{i}$ and $r_{i}$ are from (2.2)-(2.5) and $\tilde{\theta}_{i}$ and $\tilde{r}_{i}$ are their analogs when $\gamma$ there is replaced by $\gamma+\varepsilon$. Moreover, since $\bar{D} \subseteq D_{\varepsilon}$,

$$
r_{1}>\tilde{r}_{1} .
$$

We carefully spell out the $S_{\beta}$ notation in the statement of the lemma. From the lines after (6.5),

$$
S_{\beta}=\left\{w=u+i v: u>\ln \tilde{r}_{1},\left|\frac{v-\psi(u)}{\theta(u)}\right|<\frac{\beta}{\pi}\right\}
$$

(we wrote $r_{1}$ there, but that is really $\tilde{r}_{1}$ in our current notation), where

$$
\begin{aligned}
\psi(u) & =\frac{1}{2}\left[\tilde{f}_{2}\left(e^{u}\right)+\tilde{f}_{1}\left(e^{u}\right)\right], \\
\theta(u) & =\tilde{f}_{1}\left(e^{u}\right)-\tilde{f}_{2}\left(e^{u}\right) .
\end{aligned}
$$


Thus for $\alpha_{i}=\frac{\pi}{2}\left(1-\varepsilon_{i}\right), 0<\varepsilon_{i}<1$,

$$
\begin{aligned}
u+i v \in S_{\alpha_{i}} & \Leftrightarrow u>\ln \tilde{r}_{1} \text { and }-\frac{1-\varepsilon_{i}}{2} \theta(u)<v-\psi(u)<\frac{1-\varepsilon_{i}}{2} \theta(u) \\
& \Leftrightarrow u>\ln \tilde{r}_{1} \text { and } \tilde{f}_{2}\left(e^{u}\right)+\frac{\varepsilon_{i}}{2} \theta(u)<v<\tilde{f}_{1}\left(e^{u}\right)-\frac{\varepsilon_{i}}{2} \theta(u) .
\end{aligned}
$$

Also, since $F(w)=e^{w}$ (see Lemma 6.1),

$$
F^{-1}(D)=\left\{w=u+i v: u>\ln r_{1}, f_{2}\left(e^{u}\right)<v<f_{1}\left(e^{u}\right)\right\} .
$$

Hence the proof of the lemma comes down to showing there exist positive $\varepsilon_{1}, \varepsilon_{2}$ and $N$, with $\varepsilon_{2}<\varepsilon_{1}<1$, such that for $u \geq N$

$$
\begin{aligned}
& f_{2}\left(e^{u}\right)<\tilde{f}_{2}\left(e^{u}\right)+\frac{\varepsilon_{1}}{2} \theta(u), \\
& \tilde{f}_{1}\left(e^{u}\right)-\frac{\varepsilon_{1}}{2} \theta(u)<f_{1}\left(e^{u}\right), \\
& \tilde{f}_{2}\left(e^{u}\right)+\frac{\varepsilon_{2}}{2} \theta(u)<f_{2}\left(e^{u}\right), \\
& f_{1}\left(e^{u}\right)<\tilde{f}_{1}\left(e^{u}\right)-\frac{\varepsilon_{2}}{2} \theta(u) .
\end{aligned}
$$

Equivalently, we must find $0<\varepsilon_{2}<\varepsilon_{1}<1$ such that

$$
\begin{aligned}
& \frac{\varepsilon_{2}}{2}<\frac{f_{2}\left(e^{u}\right)-\tilde{f}_{2}\left(e^{u}\right)}{\theta(u)}<\frac{\varepsilon_{1}}{2}, \\
& \frac{\varepsilon_{2}}{2}<\frac{\tilde{f}_{1}\left(e^{u}\right)-f_{1}\left(e^{u}\right)}{\theta(u)}<\frac{\varepsilon_{1}}{2}
\end{aligned}
$$

for large $u$.

Set $t_{1}=r_{1}^{-1}\left(e^{u}\right)$ and $\tilde{t}_{1}=\tilde{r}_{1}^{-1}\left(e^{u}\right)$; then

$$
r_{1}\left(t_{1}\right)=\tilde{r}_{1}\left(\tilde{t}_{1}\right) .
$$

By (2.2) and its tilde analog, as $u \rightarrow \infty$

$$
\frac{t_{1}^{2}}{\tilde{t}_{1}^{2}}=\frac{1+(\gamma+\varepsilon)^{2} \widetilde{t}_{1}^{2 p-2}-2(\gamma+\varepsilon) h\left(\tilde{t}_{1}\right)}{1+\gamma^{2} t_{1}^{2 p-2}-2 \gamma h\left(t_{1}\right)} \rightarrow\left\{\begin{array}{cc}
\frac{1+(\gamma+\varepsilon)^{2}}{1+\gamma^{2}}, & p=1 \\
1, & p<1
\end{array}\right.
$$

Moreover,

$$
t_{1}^{2}-\tilde{t}_{1}^{2}=\frac{r\left(t_{1}\right)^{2}\left[(\gamma+\varepsilon)^{2} \tilde{t}_{1}^{2 p-2}-\gamma^{2} t_{1}^{2 p-2}-2\left[(\gamma+\varepsilon) h\left(\tilde{t}_{1}\right)-\gamma h\left(t_{1}\right)\right]\right]}{\left[1+(\gamma+\varepsilon)^{2} \tilde{t}_{1}^{2 p-2}-2(\gamma+\varepsilon) h\left(\tilde{t}_{1}\right)\right]\left[1+\gamma^{2} t_{1}^{2 p-2}-2 \gamma h\left(t_{1}\right)\right]} .
$$

Since

$$
r_{1}^{2}(t) \sim \begin{cases}t^{2}\left(1+\gamma^{2}\right), & p=1, \\ t^{2}, & p<1,\end{cases}
$$

as $t \rightarrow \infty$ (using (2.2) and that $h(t) \rightarrow 0$ ), we get

$$
t_{1}^{2}-\tilde{t}_{1}^{2} \sim\left\{\begin{array}{l}
\frac{t_{1}^{2}\left[2 \varepsilon \gamma+\varepsilon^{2}-2\left[(\gamma+\varepsilon) h\left(\tilde{t}_{1}\right)-\gamma h\left(t_{1}\right)\right]\right]}{\left[1+(\gamma+\varepsilon)^{2}\right]}, \\
t_{1}^{2}\left[(\gamma+\varepsilon)^{2} \tilde{t}_{1}^{2 p-2}-\gamma^{2} t_{1}^{2 p-2}-2\left[(\gamma+\varepsilon) h\left(\tilde{t}_{1}\right)-\gamma h\left(t_{1}\right)\right]\right]
\end{array}\right.
$$

as $u \rightarrow \infty$.

If $p=1$, then (8.5)

$$
\frac{t_{1}^{2}-\tilde{t}_{1}^{2}}{\tilde{t}_{1}^{p-1} t_{1}^{2}} \rightarrow \frac{2 \varepsilon \gamma+\varepsilon^{2}}{1+(\gamma+\varepsilon)^{2}} \quad \text { as } \quad u \rightarrow \infty,
$$


whereas if $p<1$, we get

$$
\begin{aligned}
& \frac{t_{1}^{2}-\tilde{t}_{2}^{2}}{\tilde{t}_{1}^{p-1} t_{1}^{2}} \\
& \quad \sim \tilde{t}_{1}^{p-1}\left[(\gamma+\varepsilon)^{2}-\gamma^{2}\left(\frac{t_{1}}{\tilde{t}_{1}}\right)^{2 p-2}-2(\gamma+\varepsilon) \tilde{t}_{1}^{2-2 p} h\left(\tilde{t}_{1}\right)-2 \gamma\left(\frac{\tilde{t}_{1}}{t_{1}}\right)^{2-2 p} t_{1}^{2-2 p} h\left(t_{1}\right)\right] \\
& \quad \rightarrow 0 \text { as } u \rightarrow \infty, \text { by (2.9) }) .
\end{aligned}
$$

In any case, the hypothesis of Lemma 3.6 holds, and the lemma yields

$$
\frac{f\left(t_{1}\right)-f\left(\tilde{t}_{1}\right)}{\tilde{t}_{1}^{p-1}} \rightarrow 0 \quad \text { as } \quad u \rightarrow \infty .
$$

Thus by (2.4) and its tilde analog, using Lemma 3.5] and (8.5),

$$
\begin{aligned}
& \frac{\tilde{f}_{1}\left(e^{u}\right)-f_{1}\left(e^{u}\right)}{\tilde{t}_{1}^{p-1}}=\frac{\tilde{\theta}_{1}\left(\tilde{t}_{1}\right)-\theta_{1}\left(t_{1}\right)}{\tilde{t}_{1}^{p-1}} \\
& =\frac{f\left(\tilde{t}_{1}\right)-f\left(t_{1}\right)}{\tilde{t}_{1}^{p-1}}+\frac{1}{\tilde{t}_{1}^{p-1}} \arccos \frac{1-(\gamma+\varepsilon) h\left(\tilde{t}_{1}\right)}{\sqrt{1+(\gamma+\varepsilon)^{2} \tilde{t}_{1}^{2 p-2}-2(\gamma+\varepsilon) h\left(\tilde{t}_{1}\right)}} \\
& \quad-\left(\frac{t_{1}}{\tilde{t}_{1}}\right)^{p-1} \frac{1}{t_{1}^{p-1}} \arccos \frac{1-\gamma h\left(t_{1}\right)}{\sqrt{1+\gamma^{2} t_{1}^{2 p-2}-2 \gamma h\left(t_{1}\right)}} \\
& \rightarrow\left\{\begin{array}{l}
0+(\gamma+\varepsilon)-\gamma, \quad 1, \quad p<1 \\
0+\arccos \frac{1}{\sqrt{1+(\gamma+\varepsilon)^{2}}}-\arccos \frac{1}{\sqrt{1+\gamma^{2}}}, \quad p=1 \text { as } u \rightarrow \infty
\end{array}\right.
\end{aligned}
$$

On the other hand, since $\tilde{r}_{1}\left(\tilde{t}_{1}\right)=e^{u}$, the tilde analog of (2.2) forces $\tilde{t}_{1} \sim e^{u}$ as $u \rightarrow \infty$, for $p<1$. Consequently

$$
\frac{\tilde{f}_{1}\left(e^{u}\right)-f_{1}\left(e^{u}\right)}{e^{u(p-1)}} \rightarrow\left\{\begin{array}{l}
\varepsilon, \quad p<1 \\
\arccos \frac{1}{\sqrt{1+(\gamma+\varepsilon)^{2}}}-\arccos \frac{1}{\sqrt{1+\gamma^{2}}}, \quad p=1
\end{array}\right.
$$

as $u \rightarrow \infty$. By Lemma 6.2 ,

$$
\frac{\tilde{f}_{1}\left(e^{u}\right)-f_{1}\left(e^{u}\right)}{\theta(u)} \rightarrow \begin{cases}\frac{\varepsilon}{2(\gamma+\varepsilon)}, & p<1 \\ \frac{\arccos \frac{1}{\sqrt{1+(\gamma+\varepsilon)^{2}}}-\arccos \frac{1}{\sqrt{1+\gamma^{2}}}}{2 \arccos \frac{1}{\sqrt{1+(\gamma+\varepsilon)^{2}}}} & p=1,\end{cases}
$$

as $u \rightarrow \infty$. In a similar way we can show that

$$
\frac{f_{2}\left(e^{u}\right)-\tilde{f}_{2}\left(e^{u}\right)}{\theta(u)} \text { converges to the same limit. }
$$

For $\varepsilon>0$ small enough, the limits are positive and small. In particular, we can choose $0<\varepsilon_{2}<\varepsilon_{1}<1$ such that (8.3)- (8.4) hold, as desired. 


\section{ACKNOWLEDGMENT}

Originally, our Theorem 1.1 gave limsup and liminf behavior, but thanks to a key suggestion by the referee, we were able to strengthen the results to the present form. We express our heartfelt thanks. We also thank Rodrigo Bañuelos for sending us copies of the papers by van den Berg and Lifshits and Shi, and we are grateful to Wenbo Li for sending us a preprint of his work.

\section{REFERENCES}

[1] Bañuelos, R. and Davis, B. (1989). Heat kernel, eigenfunctions, and conditioned Brownian motion in planar domains, Journal of Functional Analysis 84 188-200. MR.91a:60203

[2] Bañuelos, R., DeBlassie, R.D. and Smits, R. (2001). The first exit time of planar Brownian motion from the interior of a parabola, Annals of Probability 29 882-901. MR2002h:60165

[3] van den Berg, M. (2003). Subexponential behavior of the Dirichlet heat kernel, Journal of Functional Analysis 198 28-42. MR2003m:60211

[4] Li, W. (2003). The first exit time of Brownian motion from an unbounded convex domain, Annals of Probability 31 1078-1096. MR 2004c:60126

[5] Lifshits, M. and Shi, Z. (2002), The first exit time of Brownian motion from a parabolic domain, Bernoulli 8 745-765. MF 2004d:60213

[6] Spitzer, F. (1958). Some theorems concerning two-dimensional Brownian motion, Transactions of the American Mathematical Society 87 187-197. MR 21:3051

[7] Warschawski, S.E. (1942). On conformal mapping of infinite strips, Transactions of the American Mathematical Society 51 280-335. MR 4:9b

Department of Mathematics, Texas A\&M University, College Station, Texas 778433368

E-mail address: deblass@math.tamu.edu

Department of Mathematical Sciences, New Mexico State Univerity, P.O. Box 30001, Department 3MB, Las Cruces, New Mexico 88003-8001

E-mail address: rsmits@nmsu.edu 